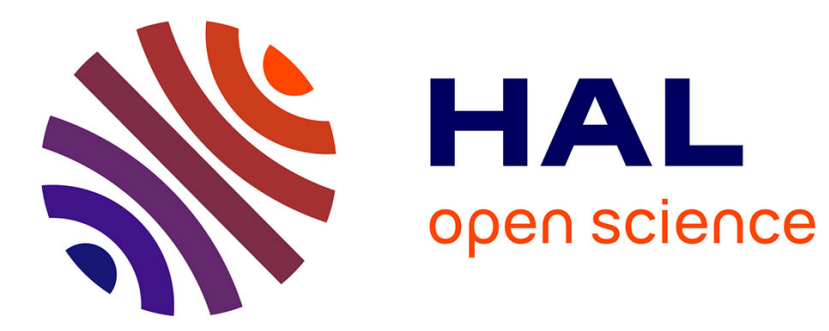

\title{
Second order division in sectors as a prepattern for sensory organs in vertebrate development
}

\author{
Vincent Fleury, Alexis Peaucelle, Anick Abourachid, Olivia Plateau
}

\section{To cite this version:}

Vincent Fleury, Alexis Peaucelle, Anick Abourachid, Olivia Plateau. Second order division in sectors as a prepattern for sensory organs in vertebrate development. Theorie in den Biowissenschaften / Theory in Biosciences, 2021, 10.1007/s12064-021-00350-w . hal-03098723v2

\section{HAL Id: hal-03098723 \\ https://hal.science/hal-03098723v2}

Submitted on 25 Nov 2021

HAL is a multi-disciplinary open access archive for the deposit and dissemination of scientific research documents, whether they are published or not. The documents may come from teaching and research institutions in France or abroad, or from public or private research centers.
L'archive ouverte pluridisciplinaire HAL, est destinée au dépôt et à la diffusion de documents scientifiques de niveau recherche, publiés ou non, émanant des établissements d'enseignement et de recherche français ou étrangers, des laboratoires publics ou privés. 


\title{
Second order division in sectors as a prepattern for sensory organs in vertebrate development
}

\author{
Vincent Fleury ${ }^{1 \#}$, Alexis Peaucelle ${ }^{2}$, Anick Abourachid ${ }^{3}$ and Olivia Plateau ${ }^{1,3,4}$ \\ ${ }^{1}$ Laboratoire Matière et Systèmes Complexes, UMR 7057 Université de Paris/CNRS, 10 rue \\ Alice Domont et Léonie Duquet, 75013 Paris, France. \\ ${ }^{2}$ Institut Jean-Pierre Bourgin, UMR 1318 INRA/CNRS \\ INRA Centre de Versailles-Grignon \\ Route de St-Cyr (RD10), 78026 Versailles Cedex, France. \\ ${ }^{3}$ Laboratoire Mécanismes Adaptatifs et Evolution, UMR 7179 MNHN/CNRS, CP 55, 57 rue \\ Cuvier 75231 Paris cedex 05, France. \\ ${ }^{4}$ Département de Géosciences, Université de Fribourg, \\ Ch. du Musée 6, 1700 Fribourg, CH \\ \#Author for correspondence, email : vincent.fleury@univ-paris-diderot.fr
}

\begin{abstract}
.
We present in vivo observations of chicken embryo development which show that the early chicken embryo presents a principal structure made out of concentric rings and a secondary structure composed of radial sectors. During development, physical forces deform the main rings into axially directed, antero-posterior tubes, while the sectors roll up to form cylinders that are perpendicular to the antero-posterior axis. As a consequence, the basic structure of the chicken embryo is a series of encased antero-posterior tubes (gut, neural tube, body envelope, amnion, chorion) decorated with smaller orifices (ear duct, eye stalk, nasal duct, gills, mouth) forming at right angles to the main body axis. We argue that the second order divisions reflect the early pattern of cell cleavage, and that the transformation of radial and orthoradial lines into a body with sensory organs is a generic biophysical mechanism more general than the chicken embryo.
\end{abstract}




\section{Introduction}

Pattern formation in biology has for long been a matter of debate. Since the pioneering ideas of D'Arcy Thomson [1] and Leduc [2] there exists a strong tradition to relate many developmental processes to generic physical processes. Even in Darwin's Origin of species, pattern formation is actually discretely ascribed to "mutual pressure" i.e. to mechanical stress gradients [3]. Although the progress in developmental biology and especially genetics, has demonstrated the causal character of genes in animal development $[4,5]$, there is a continued interest in the physical, biomechanical, aspects of animal development and evolution, since physical principles should underlie all developmental processes at some level (living material being a form of condensed matter). The view that physical processes play an important role in development has been put forward by classical embryologists such as His [6], and by such physical embryologists as Gordon [7] or Belousov [8], and others. It has been argued by Newman [9] that there exists fundamental physical kernels of development which are repeatedly used by nature to form animals. More recently, the interplay of physics and biology has started to be unravelled at a mocelular level, in the study of gastrulation [10] or of organogenesis [11], and there is now a steady flow of work on mechanotransduction [12], durotaxis [13], tensegrity [14], etc.

It is not clear though, whether the physical approach simply complements the genetic and biochemical aspects of embryo development, or whether the physical issues are so fundamental that they ideally precede animal organization. At the time of Darwin, such scholars as Owen already argued that animal forms were more fundamental than their biological understanding, and that evolution consisted in minor adjustments over general patterns, as fundamental as the orbits of the planets in astronomical science [15]. Conversely, one may argue that physical constraints, ideal forms or physical attractors are rather secondary, and that the historicity is the rule in biology [16]. While general concepts and philosphical debates have an interest and some importance, experimental work aims at finding the actual fundamental physical processes or laws, and at understanding how biochemical facts inherited from evolution may adjust or even "control", as so often stated in developmental biology, the physical variables. A clear example is the recent mathematical description of bird beak morphogenesis and evolution [16], or the celebrated work of Douady and Couder on the origin of phyllotaxis in plants [17].

Here, we will be interested on the morphogenesis of vertebrates at large, although our experimental model is the chicken embryo. The mechanism which we put forward in the sequell presents such a degree of generality, that it naturally suggests that it is at play in other taxons. This woud imply or require that inside a given animal phylum, the mechanism of formation of gross anatomical traits should follow general fundamental laws. It has long been argued that the early embryonic pattern is indeed common to all taxa, and that more specific traits appear at later stages [18]. It is true that the view inherited from Haeckel, that vertebrate embryos actually pass through a common developmental bottleneck has been 
challenged and charged with fraud [19]. However, when reading such critical point of views as Richardson's [20], one is still puzzled by the obvious similarity of embryos which are nevertheless used to reject the notion that embryos may look much alike. It seems that embryonic distance is still ill defined. Qualitative dissimilarity does not necessarily imply a great distance in terms of kinetics, or morphodynamics of the development. It is a casual fact in physics that a generic dynamic phenomenon may lead to different forms, depending on the values of physical parameters or on time scales. Small variations in parameters can imply large differences in morphogenesis. Therefore, it should not come as a surprise that embryos could still be considered as closely related even if they are not identical, if they are organized by the same dynamic phenomenon, but with shifted parameters.

If we turn to vertebrates: from the lamprey all the way to mammals, the vertebrate taxa exhibit a recognizable pattern composed of a roughly cylindrical body elongated along the Antero-Posterior (AP) axis and a series of orifices perpendicular to the main body axis (Fig. $1 \mathrm{~A}$ ), and located at the boundary between the ventral and dorsal parts of the animal (the Dorso-Ventral (DV) boundary). These orifices comprise the eyes, the nasal and otic pits and the mouth. Following our observations below, we gather together the mouth and the other sensory organs. Even the ancestors of the vertebrates, the cephalochordates and the tunicates, exhibit simultaneously a mouth and also gill slits, which are homologs to sensory organs [21]. Terrestrial vertebrates such as a chicken, are more complex than a jawless fish especially in that head flexure complicates the matter and influences organ shape [6].

The embryonic precursors of the rostral organs are called placodes [22]. Chondrichthyan vertebrates such as the sharks or rays ${ }^{1}$ display slit-like orifices, like gills, but in a similar regular pattern (Fig. 1B, C). Such slits are transiently visible in a chicken embryo (Supplementary Figure 1). The question we address in this article is that of the role of geometry and of mechanical constraints in the genesis of the chicken sensory organs, and more generally in head features. What is the topological origin of placodes and how do they dynamically transform into sensory organs in some taxa, and in slits or even jaws (Fig. 1D) in others? We rely on observations by time-Lapse (T-L) video microscopy of the developing chicken embryo to propose a fundamental physical model of sensory organ formation in this vertebrate. Our model allows us to determine theoretically how formation of the mouth and sensory organs along the body wall occurs.

We first recall how the main AP directed tubes (neural tube, body wall, amniotic sac etc.) form in a vertebrate such as the chicken. After fertilization, the first cleavages generate a concentric ring structure [23]. Cell size varies stepwise from one ring to the next (Fig. 2A). This creates a contrast of mechanical properties between the rings [24] (incidently, such rings of cells, with sizes contrast are already visible in the cephalochordate Amphioxus [21]). This contrast localizes the boundaries between body parts by a robust mechanism of foldlocking at lines of elastic discontinuity [23-25]. The main driving forces are initially distributed along the very same concentric rings [26-31]. These rings are skewed posteriorly,

\footnotetext{
${ }^{1}$ In rays, growth of a very wide pectoral flap along the Dorso-Ventral edge is a secondary process during embryogenesis.
} 
due to the additional AP asymmetry of the embryo ${ }^{2}$. This encased ring pattern contracts sequentially because the constrictions are excitable, just as a muscular reflex [28]. The importance of excitability in the very fact that embryogenesis is at all possible has been highlighted in other contexts, especially by Newman [9]. The excitable character of embryogenic contractions makes the developmental process robust against physicochemical variability : the actuator of the excitation is not quite important [28].

The main AP body features of a chicken embryo is therefore an attractor of a simple mechanical system (sequential contractions), acting on a simple reference configuration (nested rings, called a blastula), and deforming into a simple final configuration (nested tubes). The same molecular pathways used for force actuation at this early stage will be available for animal motion and muscle contraction later on in development.

We show here that the blastula presents an additional, second-order division in radial sectors. We demonstrate that buckling of the sectors drives the formation of secondary cylinders or tubes that will eventually become the sensory organs, and we give in the end a generic model of blastula deformation supporting a simple mechanistic picture of vertebrate morphogenesis.

\section{Materials and methods}

For embryos until day 3 of development, the preparation of chicken embryos for optical microscopy follows a method described in Ref. 31. Older embryos (days $>3$ ), used especially for observation of eye development, are removed from their vitelline membrane, and the head is gently taken out from the amniotic sac by aspiration with a $3 \mathrm{ml}$ plastic Pasteur pipette, which is cut at the end to have a diameter slightly greater than the diameter of the embryo body (hence a diameter of the pipette tip in the 1-2 $\mathrm{mm}$ range). Embryos can be oriented in different positions for observation by rocking them carefully with tweezers. In our optical microscopy set-up, contrast is enhanced by two-slit illumination working as a Mach-Zender type interferometer [28, 31].

Particle Imaging Velocimetry (PIV) was performed using the ImageJ software developed by Wayne Rasband at National Institute of Health, and the Tracker Plugin for ImageJ developed by Olivier Cardoso and Bérangère Abou (all plugins are freely available upon request).

Elastic modulus measurements were performed by Atomic Force Microscopy (AFM) in force spectroscopy mode. Embryos were extracted from the egg and pinned on rubber, measurements were done in a fluidic cell, in Phosphate Buffer Solution (PBS, Dubbelco). The AFM measurements were performed with a JPK nanowizard 1 AFM (JPK BioAFM Business 12489 Berlin, Germany). The cantilever used had a $2 \mu \mathrm{m}$ round bead, with a spring constant of $0.067 \mathrm{~N} / \mathrm{m}$ (NanoAndMore Spilburg Bld. A1 Steinbühlstrasse 7 D-35578 Wetzlar Germany). The maximum force applied by the cantilever was set as to perform a deformation of

\footnotetext{
${ }^{2}$ The A-P asymmetry in vertebrates stems from a polar distribution of vitellus in the oocyte, further amplified by the mechanism of sperm entry across the oocyte membrane. Here, we shall not discuss further this asymmetry.
} 
approximately $0.5 \mu \mathrm{m}$. Young modulus was determined using a simple Hertz model with the JPK data analysis tool.

All embryo observations presented here are authorized by French law R214-87 modified by the Décret n2013-118.

\section{Results}

\section{A. The blastula presents a second order radial division into sectors}

We observed the structure of the early blastodisc ${ }^{3}$ by optical microscopy. The most conspicuous structures are the encased rings already mentioned (Fig. 2A). Radial sectors are also visible (Fig. 2B, C) as from day 1 of embryo development. An anterior sector is located between 10 and 2 o'clock (12 o'clock corresponding to the anterior part of the embryo, Fig. 2B), and two symmetrical sectors located at 2-5 o'clock and 8-11 o'clock (Fig. 2C). For the sake of clarity, we make reference in the figures to Hamilton and Hamburger staging [32] ( $\mathrm{HH}$ stages). We found that the anterior sector presents further subdivisions (Fig. 2B). We present the schematic structure of the blastodisc in Fig. 2D. The radial boundaries of the sectors cross the orthoradial ring structure (Supplementary Material Fig. 2).

\section{B. The sectors transform into sensory organs}

We followed dynamically the subsequent movements of these sectors. We observed that the anterior-most sector rounds off dynamically and locks the mouth precursor (Fig. 2B, Video 1), the neighboring sector locks the eye precursors (Fig. 3A, Videos 2-4), while the next sector locks the otic (ear) territory (Fig, 3B, Videos 5-7). Detailed examination reveals that the otic territory represents only an orthoradial slice of the sectors located at 2-5 and 8-11 o'clock (Fig. 3C, see especially the last frame in Video 5, and also Supp. Material Fig. 2). We observed that the eye and the ear form respectively by rapid contractions and invaginations of the discoidal ocular (Fig. 4A, Video 8) respectively otic (Fig. 4B, Video 7, 9) placodes.

\section{The tissue comprising the sectors is excitable}

Returning to much earlier stages of development, and filming with a finer temporal resolution, we observed sporadic twitches (contractions of $15 \%$ amplitude) of the embryonic tissue already at the blastula stage, prior to actual morphogenesis, Stage HH5 (Fig. 4C, Video 10) demonstrating an intrinsic ab-initio contractility and excitability of the tissue, quite similar to that already observed for amniotic sac contraction [30, 31]. At a later stage, during neurulation and advection of the presumptive otic tissue towards the midline, we were able to observe similar sporadic contractions, which are bilateral (Fig. 4D). The pattern of contraction has a linear increase followed by a relaxation-decay. We also found that the tissue responds to electric shocks of $0.5 \mathrm{~V}$ by a rapid contraction, as we had found previously (data not shown) [31]. These observations confirm that the tissue undergoes chronic

\footnotetext{
${ }^{3}$ Called blastodisc in the case of chicken.
} 
excitable contractions during morphogenesis, whose time integral is the morphogenetic process.

\section{Involvement of neural crest cells in eye and ear formation}

The movements described above deal with the ectoderm. Ectodermal cells form an epithelial sheet of cells, and they don't migrate individually. We observe in our experiments that epithelial cells in the neural crest undergo a transition to become migrating Neural Crest Cells (NCCS) [33]. While this event is quite well documented, we are able to observe it in sufficient detail to see that NCCs migrate around the eye placode and around the ear placode (Videos 11, 12, see also Videos 7 and 9 Fig. 5A, B). The simplest interpretation is that the close contact between the placode ectoderm and the underneath neural ectoderm hinders cell migration across the placode, and NCCs simply migrate around the obstacle formed by the presumptive eyes and ears. This explains why these areas are softer, eventually, being devoid of the layer of cells which is known to differentiate into craniofacial bony structures [33]. One important observation is that the onset of NCCs migration away from the median axis correlates with a shortening and widening of the neural tube (Fig. 5C see for example Videos 12 and 13). It makes sense, that, if intercalation of cells is a mechanism for chord or neural tube convergence and extension [34], reciprocally, desintercalation of cells to form migrating cells induces a shortening and widening of the neural tube.

\section{E. Secondary tube formation occurs by buckling along lines of elastic contrast}

In our experiment, the peripheral stretch of the embryo is released when we cut the vitelline membrane. The embryo is therefore less stretched radially than physiologically. We

observed that when the incubation is started at the neurulation stage, the otic territory buckled prematurely, in a hairpin pattern forming a slit-like furrow, instead of buckling in the form of an ear (Fig. 6, Video 14). In Fig. 6 Top, the otic sector is particularly well visible (arrowhead), with the placode territory itself intersecting the sector. (If incubation is started after neurulation, the otic pit and ear form normally).

The difference in cell texture suggests a difference in mechanical properties. We performed Atomic Force Microscopy (AFM) measurements on embryos around the otic territory prior to formation of the otic placode ( $\mathrm{HH} \mathrm{10)}$, and found an elastic contrast, preceding ear formation (Fig. 7A). Visual inspection shows that the embryo is narrower and less dense in the area of the presumptive ear. When the embryo is bent with a motorized glass needle, it flexes in the area of the presumptive ear, confirming that this area is softer (Fig. 7B, Video 15).

\section{F. A sequence of contractions transforms a planar sector into a tubular secondary organ}

Both ear and eye territories undergo considerable movements during morphogenesis of the corresponding organs. Eye stalks and cups expand away from the median axis against 
the ectoderm to complete the eye placode morphogenesis (Fig. 8A) until contraction and invagination occur (Videos 16-18). In the case of the ears, the otic sector undergoes a sequence of dipolar and quadrupolar deformations. First, the sector is driven posteriorly by blastula rotation and gastrulation [26]. Next it is sheared in a hyperbolic pattern by primitive streak recession and chord extension [27]. It is then dragged towards the median axis by a dipolar pull of the neural folds [35]. (It is worth mentioning that such vortical movements have received confirmation recently [29]). Once the hairpin is positioned on the dorsal area, the ventral pull of the cardiac ring shears the ear territory anteriorly (Fig. 8A, Videos 19, 20 ; Video 20 shows a profile view of the contraction of the anterior ring, or cardiac crescent, of the blastula, showing the mechanism of head flexure). (It is worth mentioning also that the existence of such a cardiac ring has been confirmed recently [36]). Finally, the ear territory undergoes a quadrupolar contraction colinear with the DV boundary (Fig. 8C, Videos 20, 21 ; Video 21 shows the movement of quadrupolar contraction in the otic area used to extract the PIV map shown in Fig. $8 \mathrm{C}$ ). This finishes the closure of the hairpin along the ventral boundary. Eventually, a well characterized ear placode is formed. While these movements may seem very complex, they correspond to a rational sequence of quadrupolar contractions following the rings and sectors boundaries.

The main dynamic difference between ear and eye lies in the fact that the eye is formed by a double invagination of the surface epithelium and of the underlying neural epithelium (forming the lens and the eye globe), while in the ear there is a single inwards fold forming the ear pit. This difference can be traced back in Fig. 3 to the fact that the eye territory is one ring more central than the ear territory.

\section{G. Modelisation}

From all the previous observations, we conclude that the morphogenetic process is first a visco-elastic flow of tissue which contains a physical texture, characterized by patches with different elastic properties. The embryo next folds systematically along the boundaries between such domains. The folds generate tubes, either by roll up during neurulation, or by invagination during sensory organ formation. However, the contraction forces present along the domain boundaries deform considerably the texture, making the process difficult to grasp in its generality.

We may use a simple model of tissue advection in viscous flows [26], to understand very simply the mechanism of formation of otic placodes from blastodisc sectors (Fig. 9A Video 22). We start from a trapezoidal line representing the contour of one sector, intersected by one ring. The speed $\mathbf{V}$ of the tissue is given via the stream function formalism by : 
with $\mathbf{A}(x, y, z)=(0,0, \Psi(x, y))$ and with $\Psi(x, y)$ the stream function, given explicitely by 4 logarithmic vortices revolving around a hyperbolic point. This situation is what is obtained for cells pulling along the DV boundary, in a crude potential-flow hypothesis. The DV boundary is set at $y=0$, and the pull is exerted by two antagonist lines of cells located at $x=a$ and $x=c$, with a pull exerted by cells in the segment along $[-b, b]$ in Oy, pulling with opposite signs along $O x$, this gives :

$$
\Psi(x, y)=\alpha \ln \left((x-a)^{2}+(y-b)^{2}\right)-\alpha \ln \left((x-a)^{2}+(y+b)^{2}\right)-\beta \ln \left((x-c)^{2}+(y-b)^{2}\right)+\beta \ln \left((x-c)^{2}+(y+b)^{2}\right) \quad E q u .2
$$

Typical boundary movements are shown in Fig. 9A, calculated iteratively with a tabler. Symmetrical sectors, undergoing a symmetrical quadrupolar contraction, deform into a round domain. This is typical of what occurs in the anterior sector forming the mouth, where the situation is obviously symmetrical (Fig. 2B).

However, by using unequal values of $\alpha$ and $\beta$ we can introduce an AP asymmetry. This will describe lateral sectors of the body (after neurulation), pulled asymmetrically in the anterior direction. This is expected because the heart induces a forward asymmetry of the pull. While a difference in magnitude of the force creates directly an asymmetry of the movements, the asymmetry may also come from differences in visco-elastic parameters. Such asymmetries generate anything between a round placode and realistic ear-like shapes (Fig. 9B Video 23).

The purpose of this calculation is to show that a very simple quadrupolar dynamics acting on deformable territories is able to generate placodes and ear-like shapes. Since embryo morphogenesis is driven by contractions, it is not surprising that quadrupolar deformations dominate the morphogenetic process. The model predicts qualitatively that animals undergoing a more pronounced head flexure, will have more choncoidal ears in lieu of round orifices, as observed.

\section{Conclusion}

In conclusion, we have proposed a set of observations which support a minimal model of chicken embryo morphogenesis. We argue that during chicken development, the transformation of a blastodisc into a 3D rudimentary animal follows a deterministic pattern 
which is present in the chicken as early as the first day after the egg is laid. The partition into rings and sectors leads to an attractor with main tubes in the AP direction and secondary tubes in the perpendicular direction. The mechanism of formation of both tubes is similar (Fig. 9D). The presumptive organ domains are not standing waves of chemicals à la Turing [37], but an elastic excitable texture with straight boundaries that deform dynamically and round off under quadrupolar contractions. Since the secondary tubes are initially locked to radial sectors, they form at right angles to the main AP tubes. Since these tubes form as a secondary process, they are smaller, and since they form on the dorsal side of the already formed neural tube, they naturally form sensory organs.

We have observed in the experiments that if buckling occurs prematurely, the fold follows the edges of the sector and takes on a hairpin slit form, instead of forming a cylindrical tube. This gives a possible physical explanation to the transition between orificelike tubes, and gill-like slits : the buckling pattern either follows the edges of the sector, and makes a slit, or rounds off inside a trapezoidal orthoradial section of a sector, and hence pinches off an orifice. Heart shear and head flexure contribute to the formation of an asymmetrical ear by an asymmetrical quadrupolar contraction along the DV boundary, with a singular pinching of the hairpin (See Fig. 9A); this gives an explanation to the morphological difference between mere round orifices, and more convoluted ear forms, as in, say, humans. This might also give a basis to the physical separation of ears and jaws during evolution [38]. The asymmetrical model of deformation of the ear territory is a simple visco-elastic picture of the classical flexure of elastic tubes, as proposed by His [6]. It also makes use, essentially, of shear forces (either symmetrical or asymmetrical), as is the case also in mathematical modelling of bird beak development [16].

One may naturally wonder how early is the blastula texture defined. While we have not access to chicken embryos earlier than day 1, existing in vivo images of blastulas of eggs retrieved from hen oviducts prior to shell formation $[39,40]$ show a clear pattern of cell texture in rings and sectors (Fig. 10A). Especially, the formation of sectors with smaller cells in a hairpin pattern (cells are smaller in the centripetal direction following the sectors more towards the center of the blastodisc) finds a straightforward explanation when remembering the dynamics of the first cell divisions. Indeed, it is known that, in vertebrates, the first two cell divisions occur in the average blastodisc plane and generate a planar pattern of four cells. The next division is radial (Fig. 10B Right, top-left). The next cell division is 
perpendicular to the blastodisc plane and it generates the underneath layer [25]. The next rounds of divisions occur in the concavities (hairpins) of the third divisions, towards the center of the blastodisc (Fig. 10B). The greater size of the peripheral cells is ascribed classically to yolk inhibition [42]. Errera's rule [43] or physical stress equilibrium [44] both lead to a minimal (minimal elastic energy, or minimal wall area) radial and orthoradial partition of sectors during cleavage, generating a structure in concentric rings, with crisscross sectors forming hairpins (and these are also found in soap bubbles arrangements [43]). This observation suggests that the basic form of vertebrates, which consists of elongated nested tubes, decorated by perpendicular orifices diving down into the brain is a physical attractor of minimal cleavage, followed by deterministic contraction and buckling along the biaxial principle lines of the cleavage. The orthoradial lines may be viewed as circular "pursestrings", and the radial lines as radiating strings. The main feature of this process is the topological conservation of radial and orthoradial 2D boundaries, which project themselves onto axial Antero-Posterior and orthogonal Medio-Lateral 3D tubes, giving support to the existence of a vertebrate structural archetype. The mapping of the orthogonal lines onto orthogonal tubes has a flavour of a conformal mapping. The conservation of the texture during the entire morphogenetic process is possibly related to the locking of cell division dynamics onto the already existing pattern of extracellular matrix at the moment of division, as reported in cell biology studies [45], and to the very slow creeping fluid dynamics [26].

The concept of archetype has been put forward by classical embryologists and evolutionnists following Owen and Darwin, who both invoke either an ideal pattern, or a structural ancestor for animal patterns, especially vertebrates ${ }^{4}$. While it is obviously true that molecular, and especially genetic facts may orient development towards completely different animal plans, such as that of the arthropods and that of the vertebrates, nevertheless, our observations and models suggest a robust chain of development concatenating physical optimal cleavage, followed by physical visco-elastic deformation along the lines of cleavage. This generates a physical attractor for the establishment of the main structure, which might be called a dynamic archetype, if not truly an animal yet. The

\footnotetext{
${ }^{4}$ The term archetype is referred as a different concept in the main text and in the glossary of Darwin's Origin of species (1872). In the main text, it is referred as the ancestral form, while in the glossary it is referred as: "ideal primitive form upon which all the beings of a group seem to be organised" [3].
} 
difference in arthropod archetype and vertebrate archetype might be traced back to the position of the yolk in the ovocyte [23].

That a structure inherited from a radial and orthoradial texture exists in all chordates is apparent in many observed facts. Specifically, the simplest of all chordates already exhibit gills in addition to the mouth, which have a very regular order [21]. Also, "ancient" fish such as the rays have very regularly spaced radial canals and gills which function as sensory organs for feeding (rays have their eyes on top of the body, and they feed blindly) [46].

We acknowledge that the observations presented here to support the model should be complemented with in vivo measurement of stress parameters at all stages of development. Also, we did not address the question of the molecular origin of the contractions (likely actin-myosin). However, the localization of buckling events along textural boundaries and the non-linear character of folding at buckling set-points presumably render the morphogenetic process at the tissue scale physically robust against the molecular details at the microscopic scale. The description of chicken embryo development which we have given may naturally be extended to other taxons. In all embryos, a structure in rings and sectors is visible at the morula/early blastula stage. Such a structure in rings and sectors is already present in cephalochordates, and even ascidians blastulas where it is quite visible (see figures in Ref. 47); the pattern of early blastula cleavage in vertebrates has been argued to be ancestral, and among the least derived [48].

\section{Acknowledgement}

We thank Nicolas Chevalier for his interest and support, and for a careful reading of the manuscript. We thank an anonymous referee for constructive remarks and relevant references.

\section{References}

1. W. d'Arcy Thomson, On growth and Form, revised edition (Dover, New York 1992)

2. S. Leduc, La biologie synthétique, étude de biophysique, (Poinat 1912)

3. C. Darwin, On the origin of species 6 th edition, on line https://www.gutenberg.org/files/2009/2009-h/2009-h.htm

4. C. Nüsslein-Volhard, E. Wieschaus E, Nature 287 (5785), 795-801 (1980). 
5. D. Duboule, Guidebook to the Homeobox Genes, (Oxford University Press, Oxford 1994).

6. W. His, Unsere Körperform und das physiologische Problem ihrer Entstehung. Briefe an einen befreundeten Naturforscher (Engelmann, Leipzig 1874).

7. N. K. Gordon and R. Gordon, Embryogenesis Explained, World Scientific, (Singapore 2016)

8. A. S. Ermakov, Biosystems, 2018 Nov;173:26-35.

doi: 10.1016/j.biosystems.2018.10.010. Epub 2018 Oct 10.

9. S. Newman, Science. 338 (6104): 217-219. (2012)

10. E. Farge, Curr. Biol., 13, 1365-1377 (2003).

11. F. Le Noble, D. Moyon, L. Pardanaud, L. Yuan, V. Djonov, R. Mattheijssen, C. Bréant, V. Fleury, A. Eichmann, Development, 131, 361-37 (2004).

12. E. K. Paluch, C. M. Nelson, N. Biais, B. Fabry, J. Moeller, B. L. Pruitt, C. Wollnik, G. Kudryasheva, F. Rehfeldt, and W. Federle, BMC Biol. 13: 47, (2015).

13. F. Alisafaei, X. Chen, T. Leahy, P. A. Janmey and V. B. Shenoy, Soft Matter, 2, (2021)

14. D. E. Ingber, J. of Cell Sci. 116,1157-1173 (2003). doi: 10.1242/jcs.00359

15. N. A. Rupke, Isis, 84, (2) 21-255 (1993).

16. O. Campas, R. Mallarino, A. Herrel, A. Abzhanov, and M. P. Brenner, PNAS 107 (8) (2010).

17. S. Douady and Y. Couder, PRL 68, 2098 (1992).

18. K. E. von Baer, Über Entwickelungsgeschichte der Thiere (Ludwig Stieda , Königsberg 1828).

19. N. Hopwood, Haeckel's Embryos: Images, Evolution, and Fraud (University of Chicago Press, Chicago, 2015).

20. M. K. Richardson, J. Hanken, M. L. Gooneratne, C. Pieau, A. Raynaud, L. Selwood, G. M. Wright, Anat. Embryol. 196, 91-106 (1997).

21. C. V. H. Baker, M. Bronner-Fraser, Dev. Biol. 232, 1-61 (2001).

22. E. Carvalho, F. Lahaye, L. Wen Yong and J. C. Croce, H. Escriv, J. Yu and M. Schubert, bioRxiv, 2020, doi: https://doi.org/10.1101/2020.05.26.112193.

23. V. Fleury, Chaos, Solitons and Fractals, 105, 230-234, (2017).

24. V. Fleury, N. Chevalier, F. Furfaro, J.-L. Duband, Eu. Phys. J. E 38, (6), 1-19 (2015).

25. G. W. Brodland, D. I.-L. Chen, J. H. Veldhuis, Int. J. Plastic. 22, 965 (2006). 
25. D. A. Voronov and L. A. Taber, Dev. Dyn. 224, 413 (2002).

26. V. Fleury, Organogenesis, 2, 16-16 (2005).

27. V. Fleury, Biosystems, Special issue “Morphogenesis", 109, 460-474 (2012).

28. V. Fleury, A. V. Murukutla, A., N. Chevalier, B. Gallois, B., M. Capellazzi-Resta, P. Picquet, Peaucelle, Phys. Rev. E 94, 022426-022444 (2016).

29. G. Shah, K. Thierbach, B. Schmid et al., Nature Comm. 10, 5753 (2019).

30. N. Tipping and D. Wilson, Anat. Rec. (Hoboken) 294, 1143 (2011).

31. V. Fleury, A. V. Murukutla, Eu. Phys. J. E. 42, 104 (2019) doi:10.1140/epje/i2019-11869-8.

32. V. Hamburger, H. L. Hamilton, Journal of Morphology 88 (1), 49-92 (1951).

33. H. Etchevers, E. Dupin, N. Le Douarin, Development 146: dev169821 (2019).

34. R. Keller, Science, 298, (5600), 1950-1954 (2002). DOI: 10.1126/science.1079478.

35. V. Fleury, Eu. Phys. J. App. Phys. 45, 30101 (2009).

36. R. Asai, Y. Haneda, D. Seya, Y. Arima, K. Fukda, Y. Kurihara, S. Miyagawa-Tomita, H.

Kurihara, Sci. Rep. 7, 8955 (2017).

37. A. M. Turing, Phil. Trans. Roy. Soc. London B 237 (641), 37-72 (1952).

38. F. Mao, Y. Hu, C. Li, Wang, M. H. A. Chase, A. K Smith, J. Meng, Science 367 (6475) 305308 (2020).

39. H. Nagai, M. Sezaki, K. Kakiguchi, Y. Nakaya, H. Ch. Lee, R. Ladher, T. Sasanami, J. Y. Han, S. Yonemura, G. Sheng, Development 142, 1279-1286 (2015).

40. H. C. Lee, H. J. Choi, T. S. Park, S. I. Lee, Y. M. Kim, S. Rengaraj, H. Nagai, G. Sheng, J. M. Lim, J. Y. Han, PLoS ONE 8 (11), e80631, (2016).

41. G. Forgács, S. A. Newman, Biological physics of the developing embryo, (Cambridge University Press 2005).

42. E. B. Wilson, The Cell and development in heredity, Third edition (Macmillan, New York, 1928).

43. S. Besson and J. Dumais, Proc. Nat. Acad. Sci., 108 (15), 6294-6299 (2011).

44. M. Louveaux, J. D. Julien, V. Mirabe, A. Boudaoud, O. Hamant, Proc. Nat. Acad. Sci., 26, 113 (30), E4294-303 (2016).

45. M. Théry, V. Racine, A. Pépin, M. Piel, Y. Chen and M. Bornens, Nat. Cell. Biol. 7 (10), 947-53 (2005).

46. L. K. Jordan, J. Morph. 269 (11), 1325-39 (2008).

47. H. Nishida, Dev. Dyn. 233, 1177-1193 (2005). 
48. J. W. Valentine, PNAS 94 (15) 8001-8005 (1997).

\section{Supplementary Material : Videos}

\section{All videos, except 15-17, are available in HD format (1600x1200), and generally with a temporal basis of $0.05 \mathrm{~Hz}(20 \mathrm{sec}$. per frame)}

Video 1 Time-Lapse of mouth sector deformation (Mag. 4X). The Video shows the onset of constriction of the entire anterior sector. It is observed that there exists a secondary smaller sector located exactly at 12 o'clock, which constricts to form the circular mouth precursor (stomodeum).

Video 2 Time-Lapse of eye sector deformation (Mag. 4X). This Video shows early stages of deformation of the anterior sector, starting from a completely flat blastula, until early neurulation. The corner of the sector is projected onto the bulge on the neural tube which will correspond to the ocular territory (Duration $8 \mathrm{Hrs}$ ).

Video 3 Time-Lapse of eye formation (Mag. 4X). This Video shows the formation of the ocular cupule and eye stalk (hammer-like tissue in the anterior part of the embryo). By the end of the Video, the flow of NCCs flowing away from the median axis is visible. (Duration 12Hrs)

Video 4 Time-Lapse of eye formation. The presumptive eye territory is found exactly at the corner of the cardiac crescent, intersecting the neural plate territory (Mag. 4X, Duration 15hrs).

Video 5 Time-Lapse of ear sector formation (Mag. 4X). The Video shows a topographical view of the embryo during neurulation, prior to ear placode formation and invagination. The presumptive placode territory appears as the apex of a sector advected towards the median axis by the movements (see Fig. 3B).

Video 6 Time-Lapse of ear formation (Mag. 4X-10X-4X). This Video shows the morphogenesis of the ear, from a state when nothing is visible in the presumptive ear territory until a well formed asymmetrical ear pit, quite reminiscent of an actual ear, is observed. The magnification was increased to 10X during placode buckling (same embryo). The heart beat in the background is a fair proof that the embryo is developing well. The flow of NCCs around the ear pit is well visible.

Video 7 Time-Lapse of ear formation (Mag. 4X). Extracted from the raw data of Video 6, the formation of the otic pit. One can see the NCCs flow around the ear ridge. The ear ridge takes on a typical quadrupolar shape because of a constriction along the DV boundary.

Video 8 Time-Lapse of eye ball formation (Mag. 10X). The Video shows the formation of the eye ball and of the lens at the highest possible magnification in our set-up (this is a direct optical microscopy, without staining, nor fluorescence). The video is available with a temporal resolution of $20 \mathrm{Sec}$. The HD resolution is $1600 \times 1200$. The Video is sharp enough to follow by PIV the initial constriction and the ectoderm invagination (see Fig. 4A). 
Video 9 Time-Lapse of otic pit formation (Mag. 10X). The Video shows the formation of the ear pit. The hairpin of tissue surrounding the ear pit during buckling is well visible. Cells are seen to register along the edge of the fold.

Video 10 Time-Lapse of a sporadic twitch (Mag. 10X). The video shows one sporadic twitch in the otic territory, much earlier than actual ear morphogenesis. The tissue is actually contractile and excitable ab initio.

Video 11 Time-Lapse of NCCs flow around eyes (Mag. 4X). This 3/4 view of neurulation shows the onset of NCCs flow, and how cells swerve around the ocular placode, as the eye stalk flattens against the surface ectoderm. Cells in the ectoderm are actually well resolved.

Video 12 Time-Lapse of NCCs flow around ears (Mag. 10X)

Video 13 Time-Lapse of cranial NCCs flow (Mag. 4X)

Video 14 Time-Lapse with hairpin buckling (Mag. 4X). In our experiments, it is often observed that the embryo buckles in a hairpin pattern converging towards the ear territory. We ascribe this effect to the fact that the embryo, detached from the vitelline membrane, is less stretched peripherally than normal. The buckling pattern reveals that there exists a centrifugal pattern with sharp latent boundaries ready to buckle.

Video 15 Head flexure experiment (two examples). The head of the embryo is gently pulled with a glass pipette fixed on a motorized stage. Flexure shows that the embryo is softer in the area of the presumptive ears.

Video 16 Time-Lapse of eye-stalk expansion. The eye stalk collides against the surface ectoderm. The collision rounds off the ocular placode; the circular territory will provide the boundary condition for ectoderm invagination (Mag. 4X)

Video 17 Time-Lapse of eye formation, at intermediate resolution (Mag. 4X). This is a frontal view of eye formation showing in cross-section the inwards buckling of the surface ectoderm. Such points of view are difficult to obtain in the chicken : the embryo is left to float in $5 \mathrm{~mm}$ of Phosphate Buffer Solution, and gently turned with tweezers in this position. The embryo is observed in an upright microscope, so that the embryo is actually resting on its back (inverted position as compared to the physiological situation). (Duration 3 hours). (Mag. 2X)

Video 18 Time-Lapse of eye formation, at low resolution (Mag. 1X). This a very low resolution TimeLapse, obtained with a binocular, showing the entire embryo. Especially, one can observe the inwards buckling of the eye ectoderm, but also the otic area. The otic pit forms along the D-V boundary. One can see the amniotic sac wrapping the head. This explains the difficulty to image correctly eye development in vivo, in the chicken model. One needs to take the head out of the amniotic sac (see text).

Video 19 Time-Lapse of heart pull (Mag. 4X). This a ventral view showing in Time-Lapse the contraction of the cardiac crescent, and the body edge. The cardiac crescent constricts and progressively twists the heart territory. The contraction of the cardiac crescent creates a purse string which pulls and shears the otic territory. 
Video 20 Time-Lapse of profile view of the embryo (Mag. $2 X$ ). This is a profile view of embryo neurulation at mag $2 X$ (followed by $4 \mathrm{X}$ ). Duration 8 hours. The film shows the ventral pull which flexes the head anteriorly.

Video 21 Quadrupolar contraction. (Mag. 10X). This brief video shows the pattern of quadrupolar contraction in the otic territory associated to head flexure, and used to calculate the PIV map of movement.

Video 22 Placode simulation, with a symmetrical quadrupole. This animation shows the advection of a sector of wedge in a symmetrical quadrupolar pull.

Video 23 Ear simulation, with a strongly asymmetrical quadrupole. This animation shows the advection of a sector of wedge in an asymmetrical quadrupolar pull. 


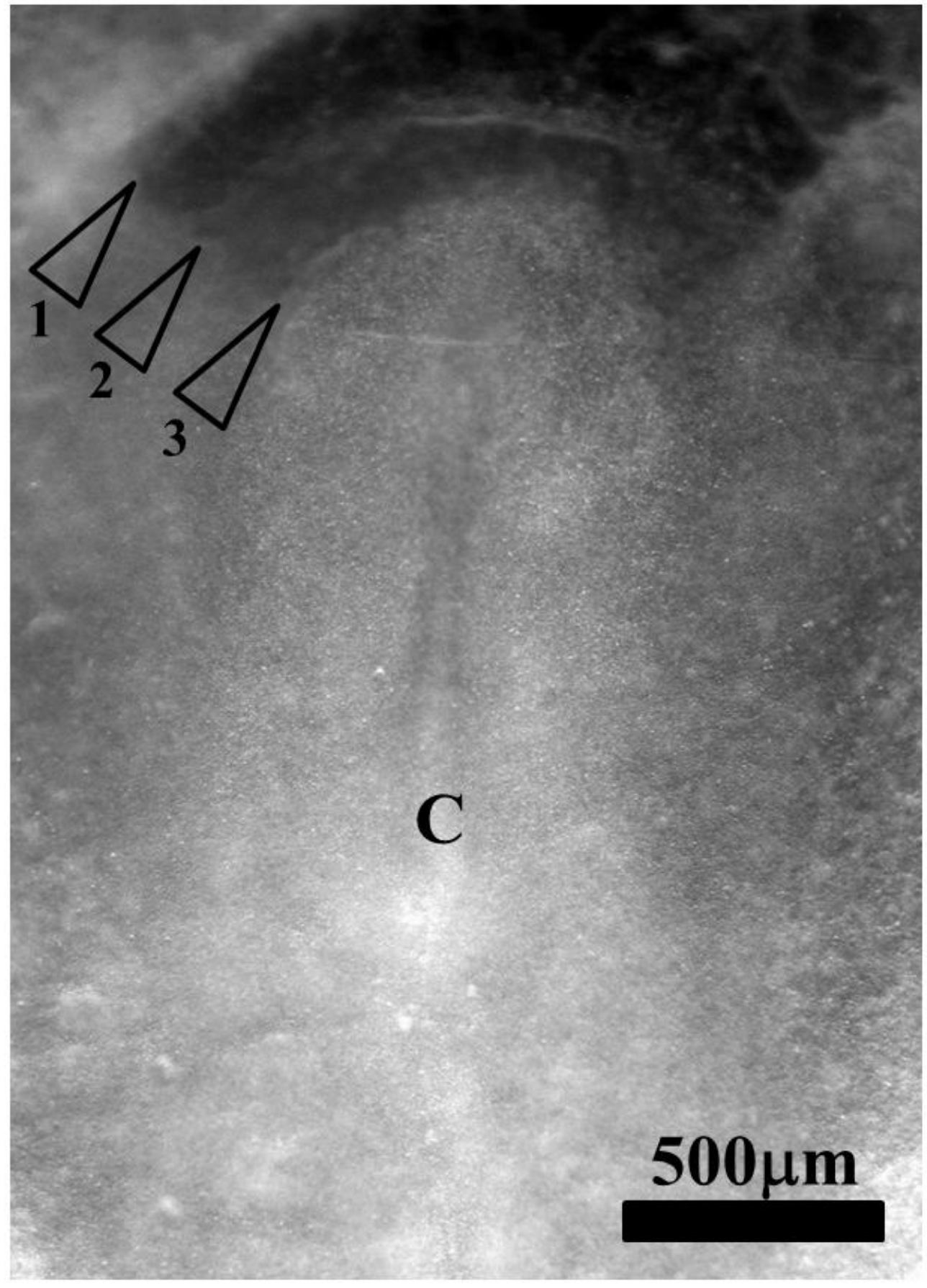

Supp. Mat. Figure 1 An embryo in which the structure in rings with sectors is exceptionally visible in the anterior part of the embryo. The three arrowheads point to the (1) amniotic ring, (2) the body envelope ring and (3) the neural ring. The visible sector line corresponds to the boundary of the ocular territory. One important observation is that sector lines cross the division in sectors. The pattern is visible without any staining of any sort, just by a difference in optical density 

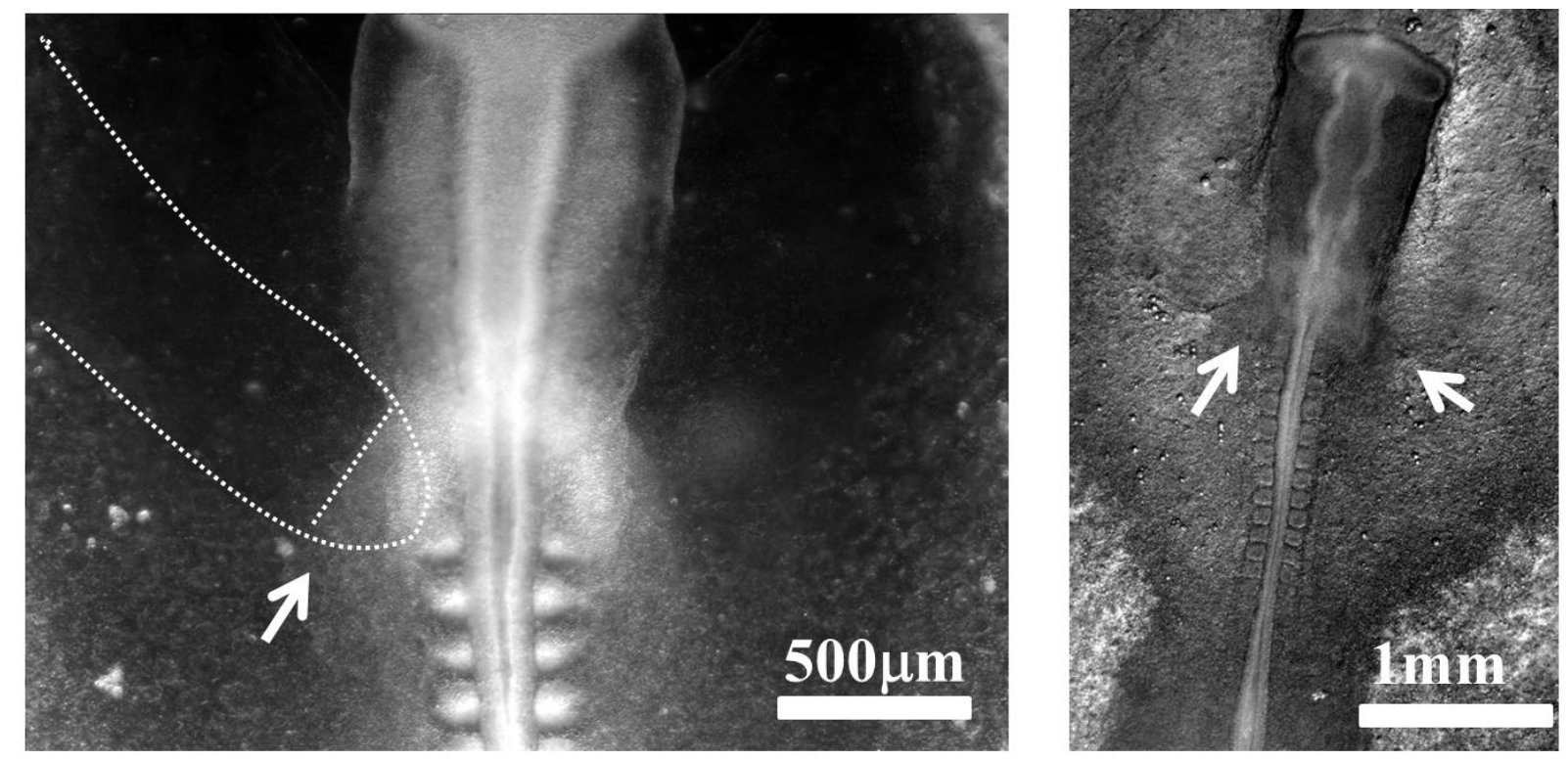

Supp. Mat. Figure 2 Left: Snapshot of an embryo during roll up of the embryo body in the area of the presumptive ear territory observed by optical microscopy (Mag. 4X). Right, last frame in Video 5 showing the two sectors on either side of the embryo body. The dorsal area is almost formed, but the lateral tissue is still lagging in plane. The otic territory appears as a slice of a sector which continues in the extra-embryonic territory. 


\section{Figures and Figure Captions}

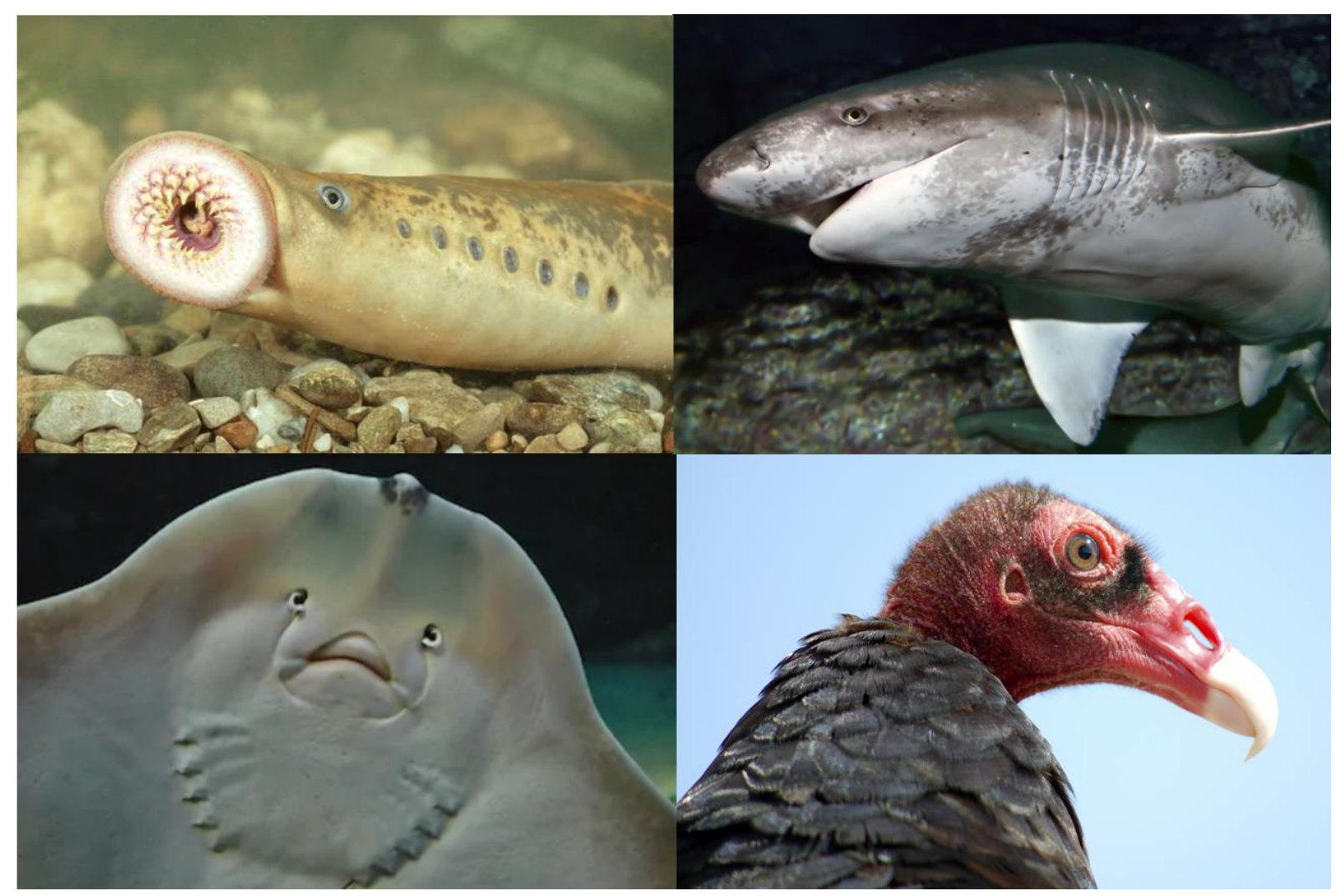

Figure 1

Figure 1 Four animals, from ancestral lampreys to sharks, stingrays and birds. The ancestral archetype is that of a tubular animal (Top-Left, the lamprey), with orifices located perpendicularly to the main axis; we count the eyes as such orifices; the mouth is a special case in that it is colinear with the tubes axis due to its position at one end. In fish, the lateral orifices may be instead slits, as in sharks (Top-Right), or organs in between slits and orifices, as in stingrays (Bottom-Left). Please note that the orifices on the sides of the stingray mouth are the ears. Stingrays have their eyes on the dorsal side, their ventral sensory organs help them capture and eat preys they can't see. In amniotes (Bottom Right), there is an additional head flexure, and the ear orifice is pinched at its Dorso-Ventral boundary, with a slit-like feature detached from the ear and contributing to jaw and gum formation. (Photo Lamprey : Blinkwinkel/Alamy, Stingray : Jorge Felix Costa, Turkey Vulture : Daniel Gleason at Cascades Raptor Center Eugene, Oregon, Shark : Monterey Bay Aquarium). 

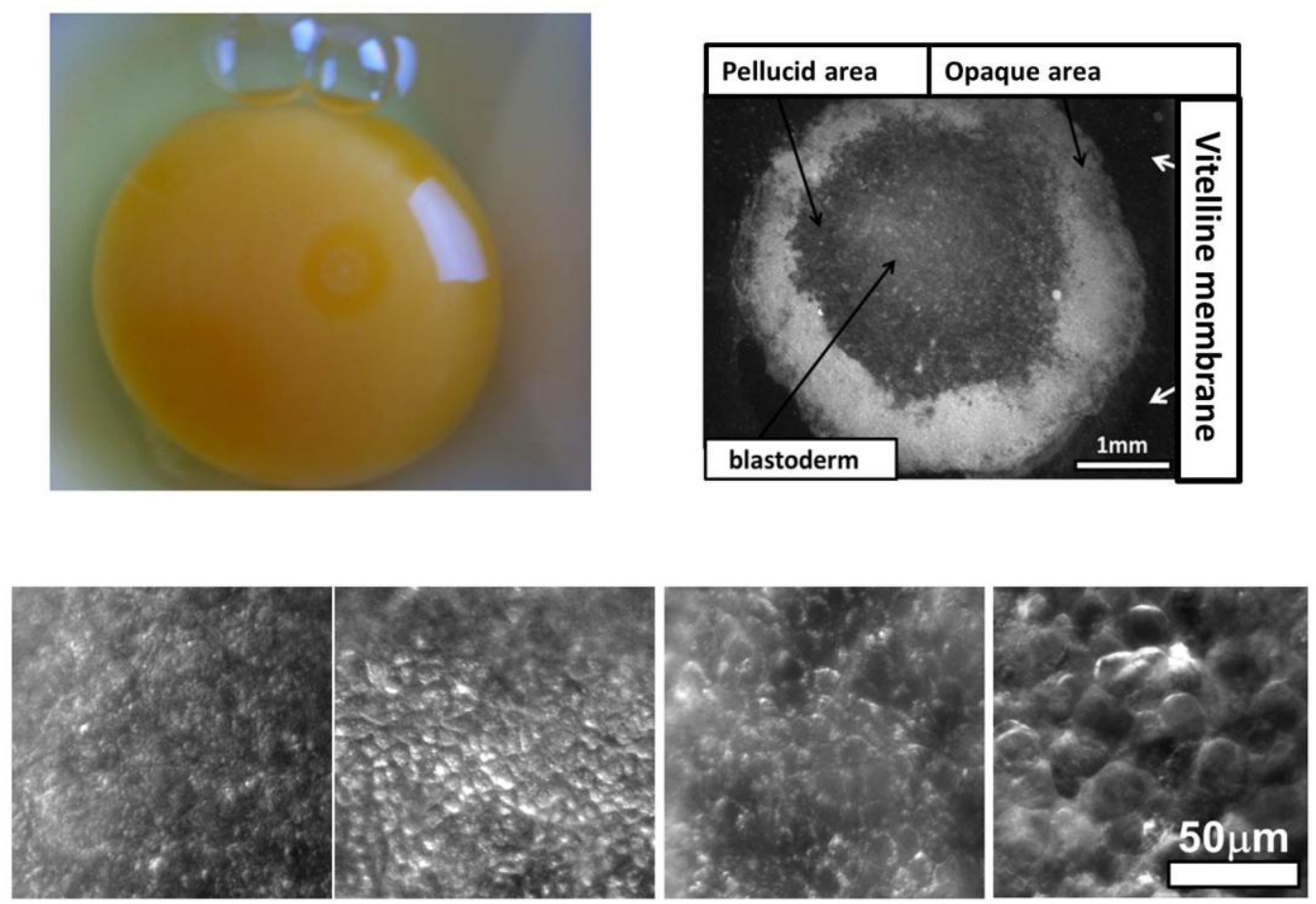

Figure 2A
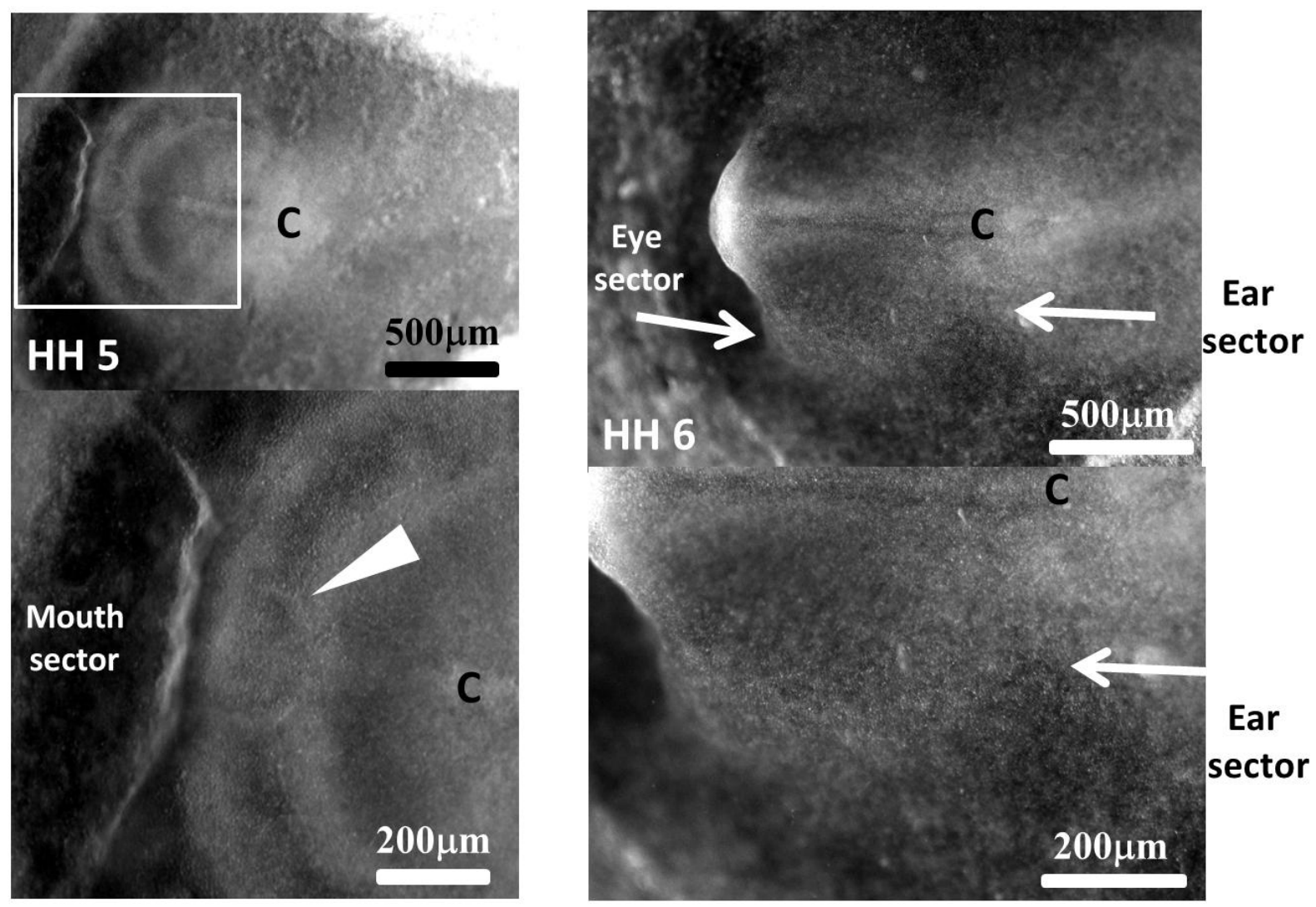
Figure 2B
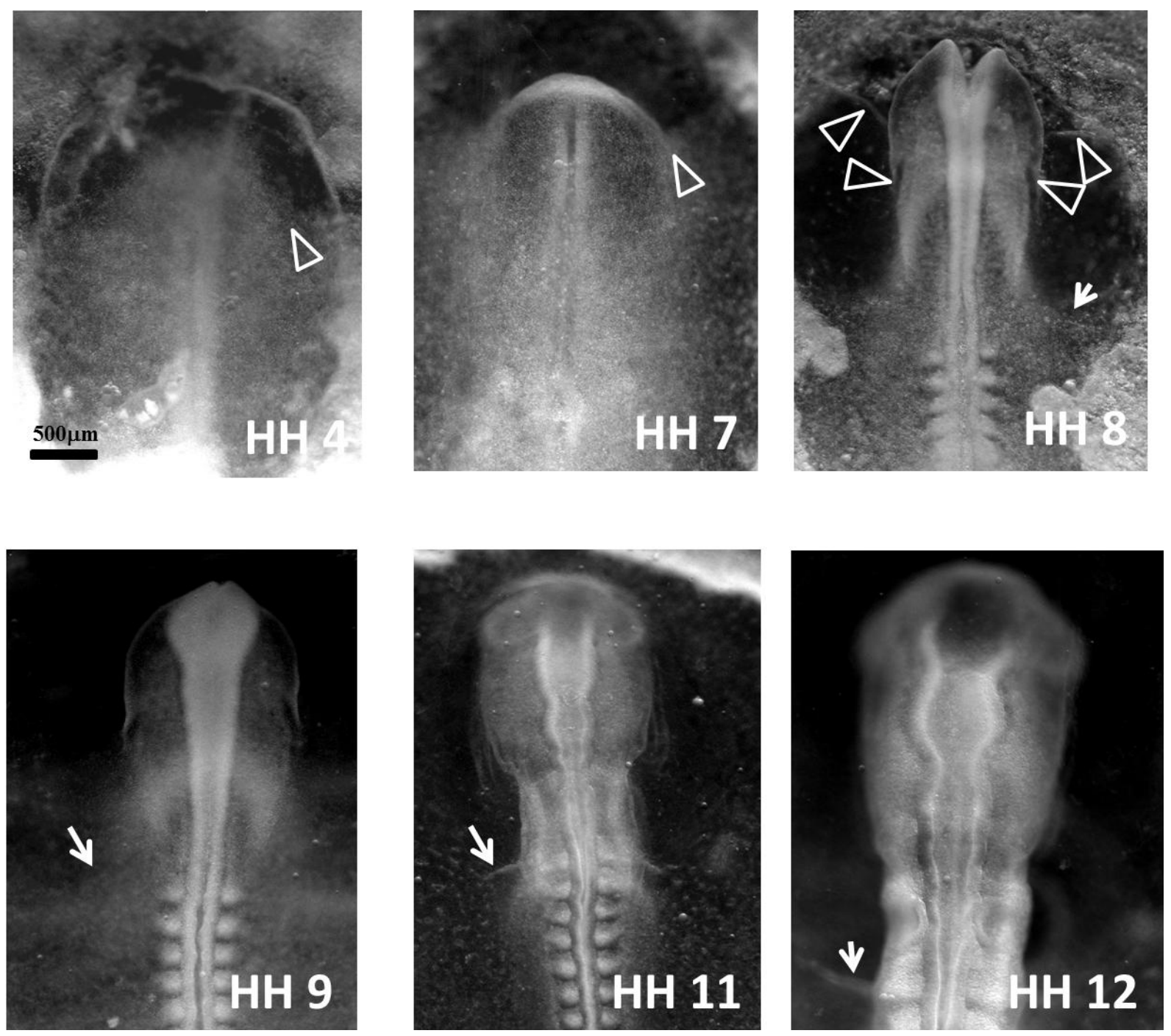

Figure 2C 


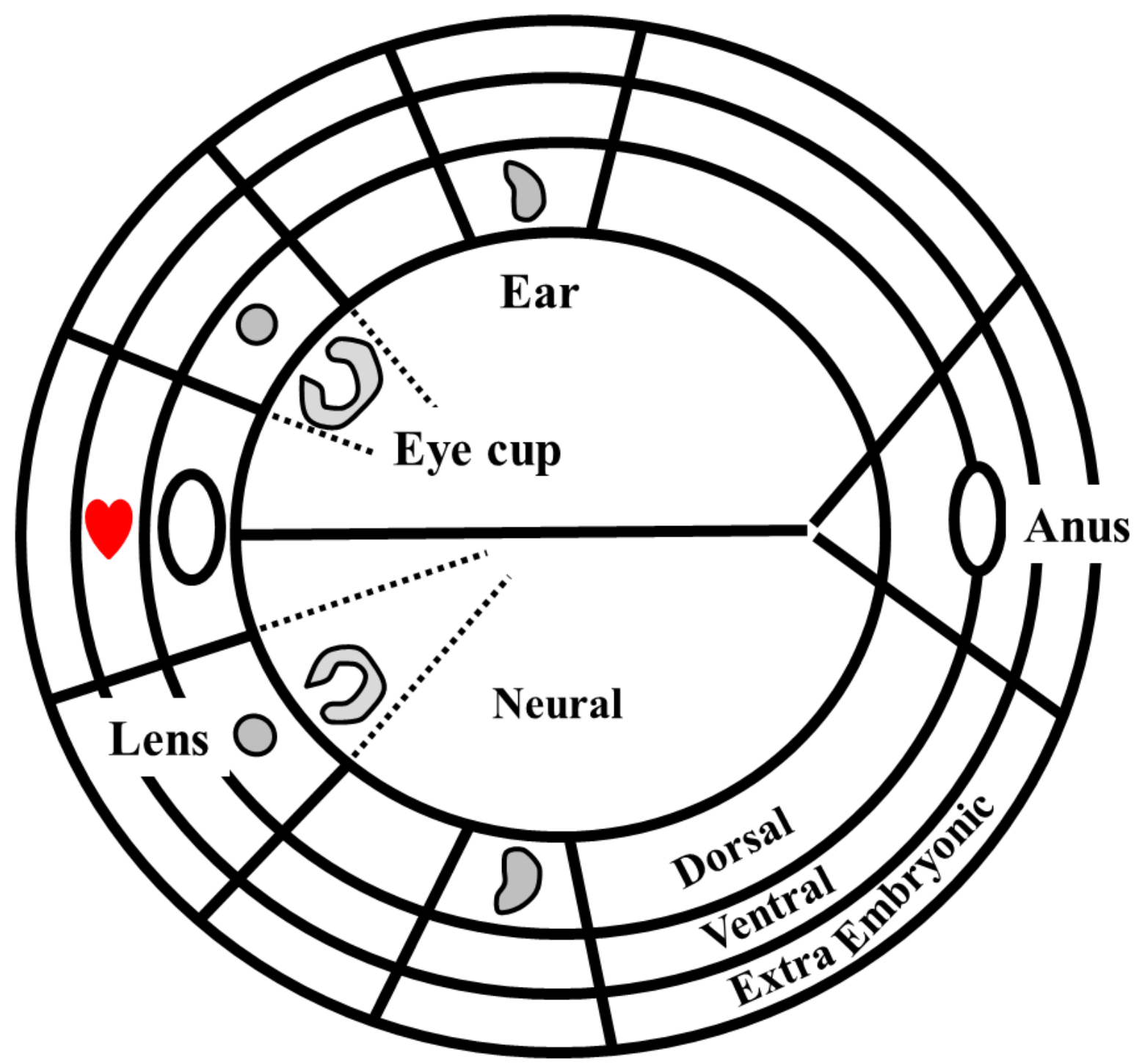

Figure 2D

Figure 2 Fig. 2A (Reprinted from Ref. 5). Optical microscopy of carefully rinsed blastulas (HH 1) show a textural pattern in rings, corresponding to the presumptive territories of the different body compartments. The rings are associated to different cell sizes, central cells being larger than peripheral cells. Fig. 2B In the next hours, a secondary structure in sectors becomes clearly visible (at earlier stages gastrulation movements render images more noisy). One anterior sector will correspond to the mouth territory (Fig. 2B Left). The arrowhead points to the stomodeum (mouth precursor, arrowhead in Fig. 2B), forming in front of the chord $(C)$ and locked by the most anterior sector. Fig. 2B Right : One next sector corresponds to the presumptive eye territory and the next sector corresponds to the ear 
territory (arrows). Fig. 2C, during the second and third day of development, the territories deform and the relationship between parts becomes more remote, but the boundaries become actually more visible. The eyes correspond to the apex of the anterior sector (arrowheads), and the ears correspond to the apex of the second sector. The continuity between the boundaries of the extraembryonic territories and the boundary of the ear itself is visible in the bottom-right image (arrows). Fig. 2D Scheme of the blastula prepattern. There exists a structure in rings corresponding in centrifugal order to neural territory, dorsal territory, ventral territory, and extra-embryonic organs. These rings are intersected by a structure in sectors. Trapezoidal territories limited by intersection of rings and sectors correspond to presumptive mouth, eyes (lens and eye ball) and ears territories which round off during morphogenesis (the nasal area was not investigated in this study). One sector also corresponds to the cardiac territory.

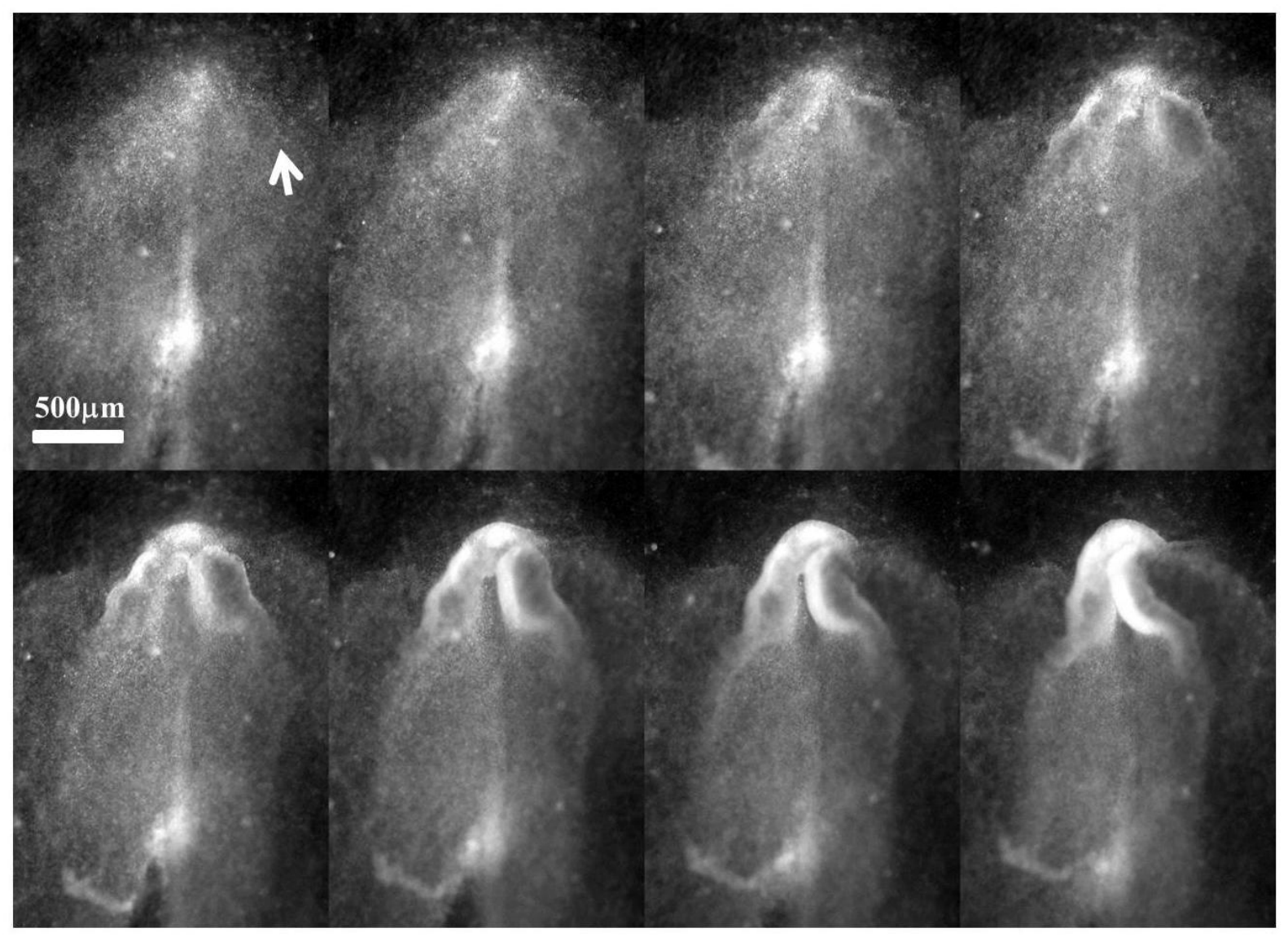

Figure 3A (Top) 


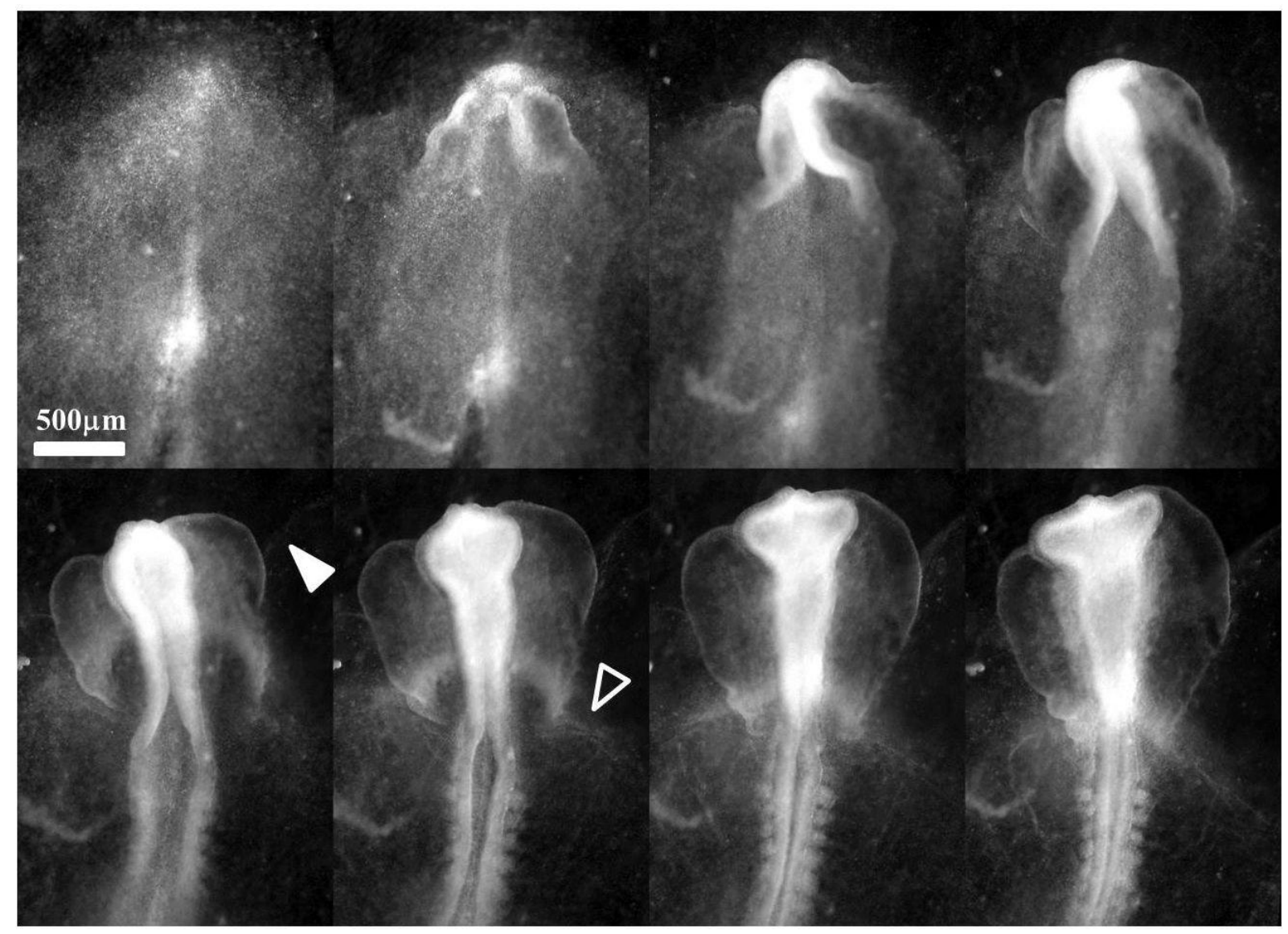

Figure 3A (Bottom) 


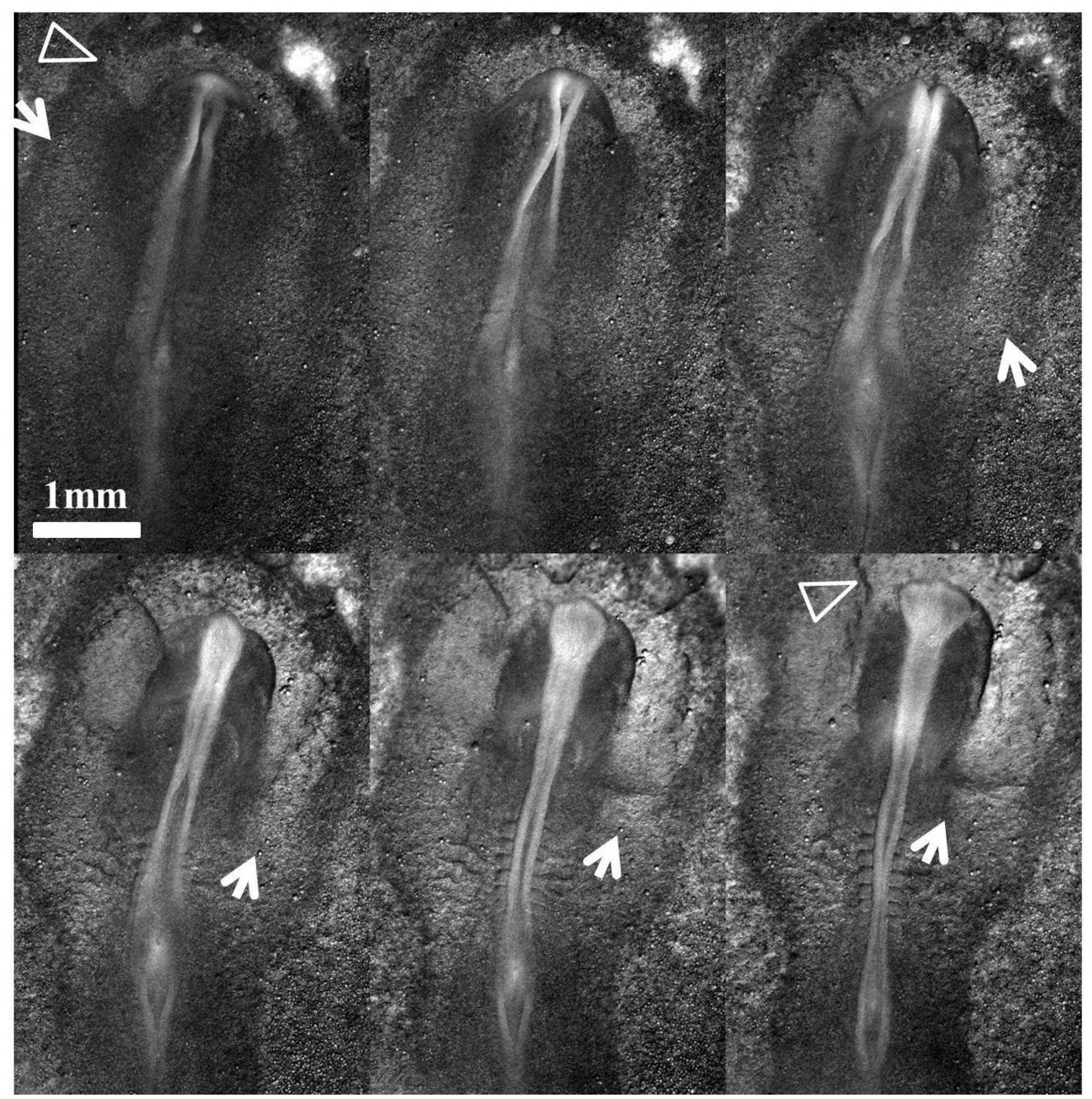

Figure 3B (Top) 


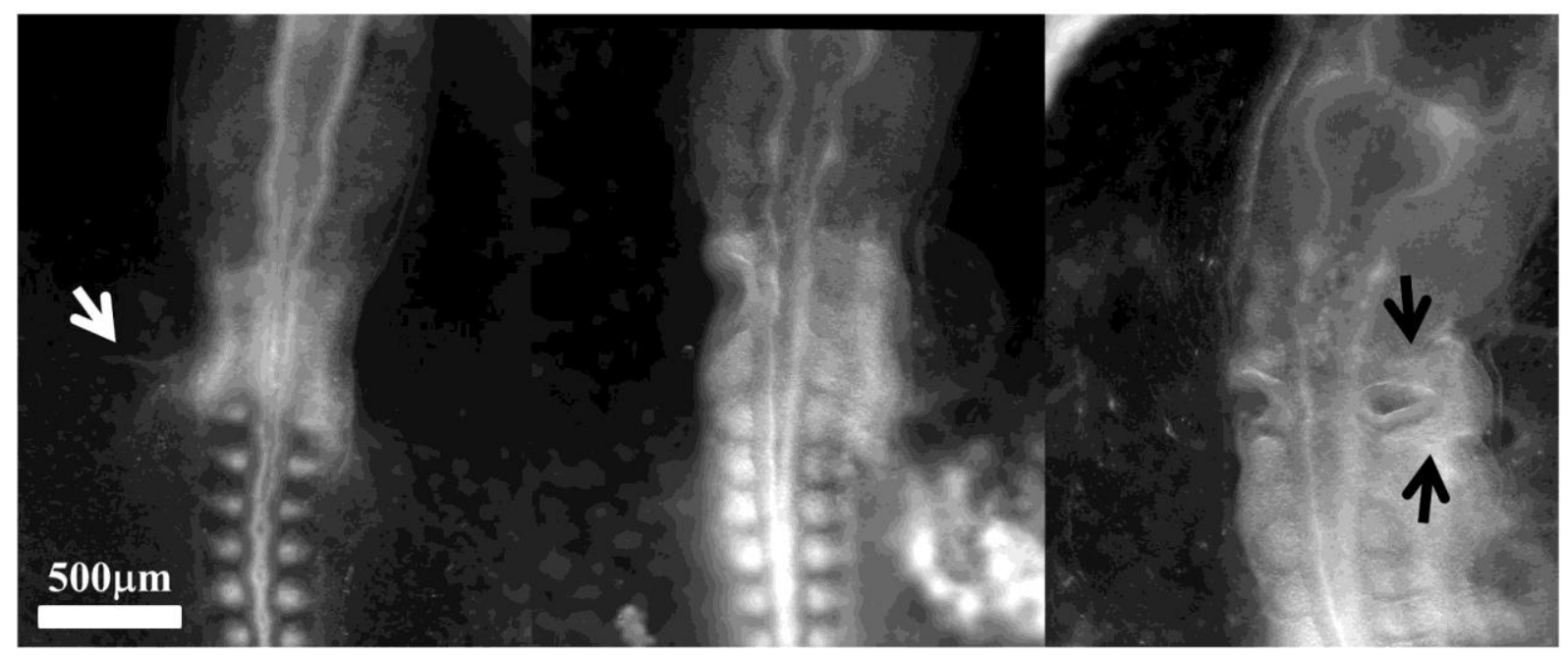

Figure 3B (Bottom)

Figure 3 Fig. 3A.The top mosaic shows the early stages of Time-Lapse in Video 5 (HH5); the second mosaic shows the entire Time-Lapse ( $\mathrm{HH}$ stages from 5 to 10) up to expansion of the eye stalk (eye stalk expansion is discussed in Ref. 35 ). The video shows that the presumptive eye territory is exactly the corner in the anterior sector of the blastula sector (arrow). This is also quite visible in Videos 2 and 3. The boundary of this sector remains visible in the embryo as a thin membranous ectodermal flap homolog to the cheek wrinkle. The sector continues away towards the extra-embryonic organs where it localizes the thinner part of the pellucid area, where the boundary of the sector remains visible (arrowhead). Fig. 3B The next sector localizes the ears. The top mosaic shows the T-L (from Video 5) of the transition from a roughly horizontal blastula to the roll up of the embryo body ( $\mathrm{HH}$ stages from 6 to 8). Again we observe sharp boundaries in the entire blastula, the anterior sector (arrowhead) corresponds to the already evoked ocular sector, while a second sector (arrows) is advected towards the neck and corresponds to the presumptive otic territory. The sectors span the entire blastula and are visible in the extraembryonic territory where they form larger portions. The bottom images (from Video 6) show three snapshots (same embryo) of ear formation at the apex of the sector which now finds itself advected on top of the neural tube (HH stages from 10 to 13). The black arrows in the last image point to the edges of the hairpin in which the otic pit has buckled downwards towards the neural tube. Please note that there is a reproducible temporal delay between left and right ear formation. Buckling 
proceeds more rapidly on the right side (to the left in Fig. 3B Bottom Right, since the embryo lies ventrally and is observed dorsally).

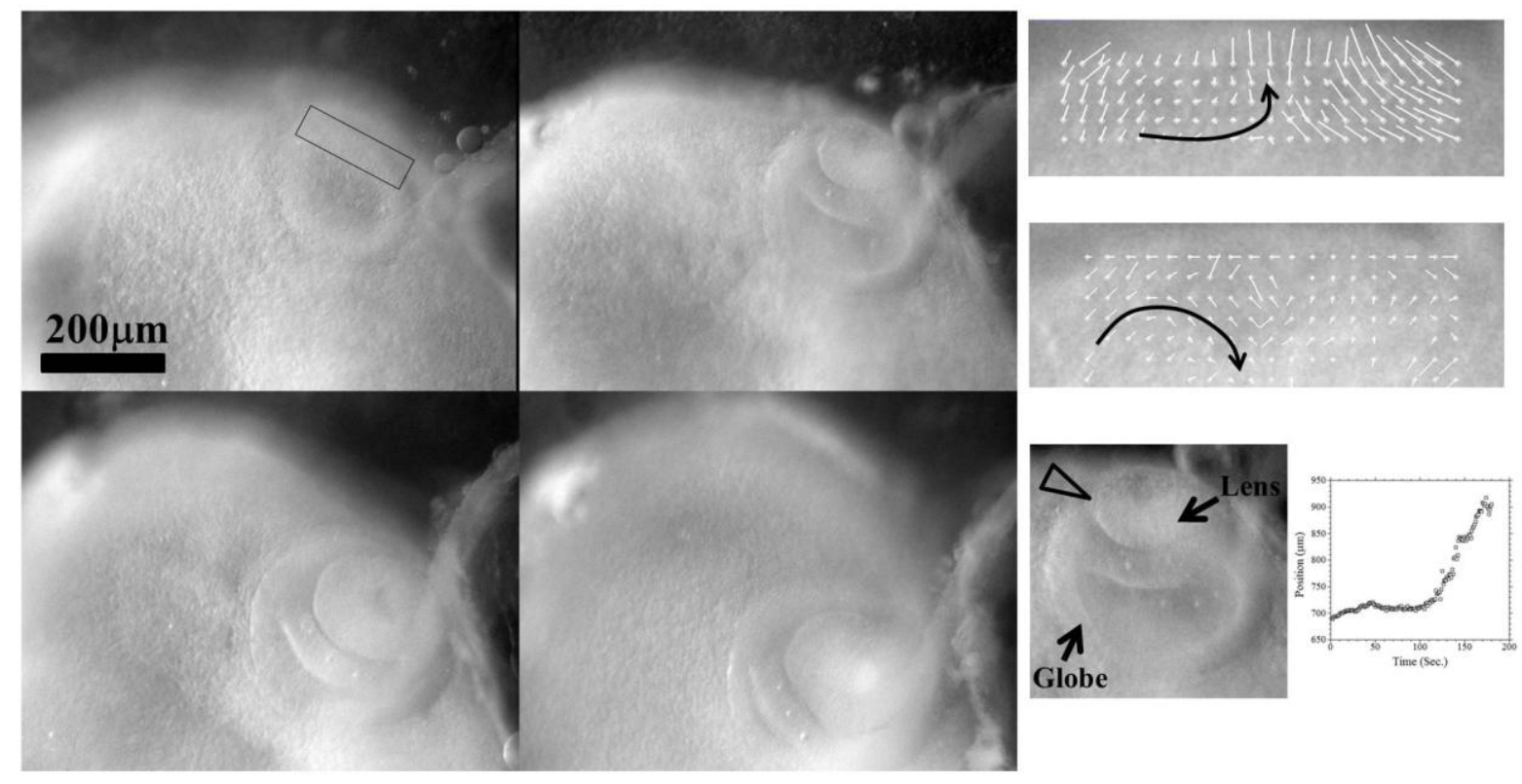

Figure 4A 


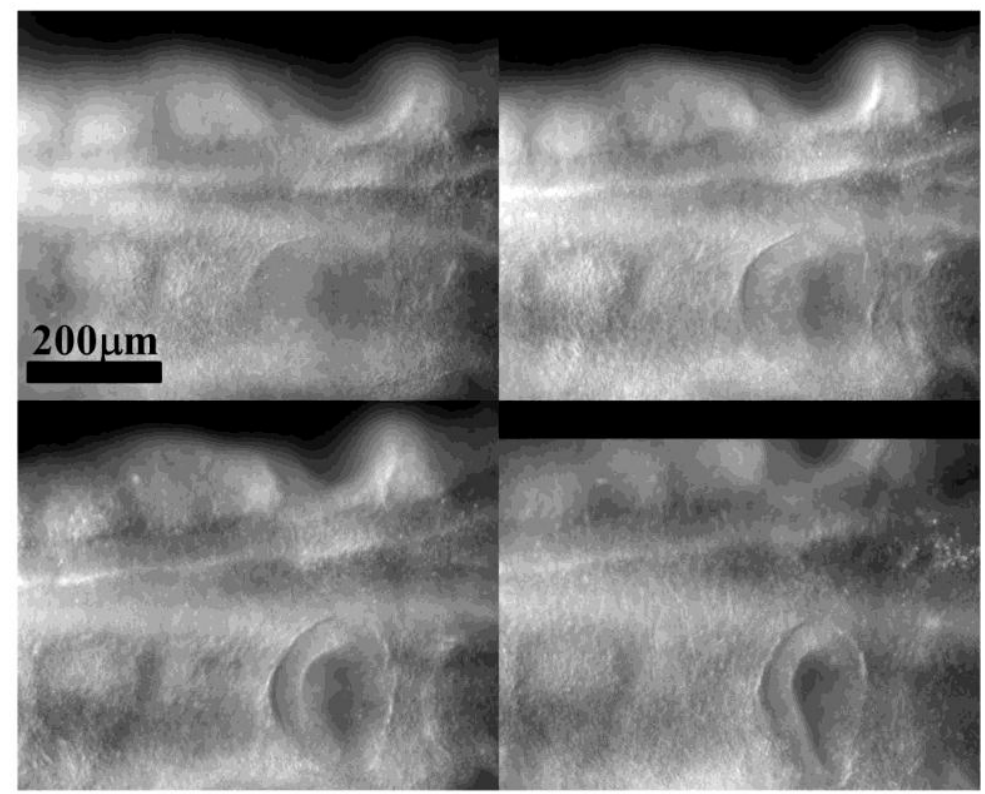

Figure 4B 

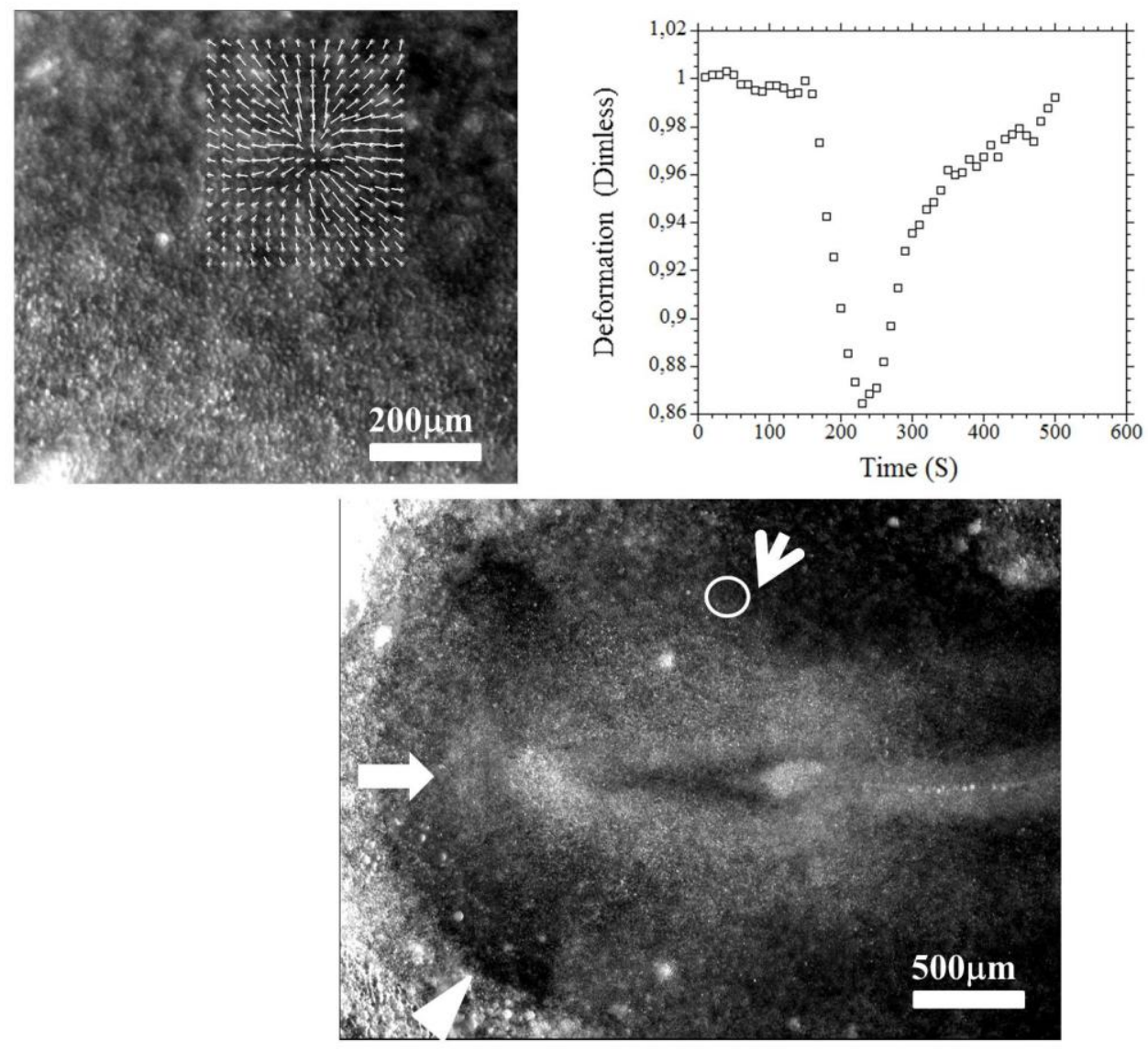

Figure 4C
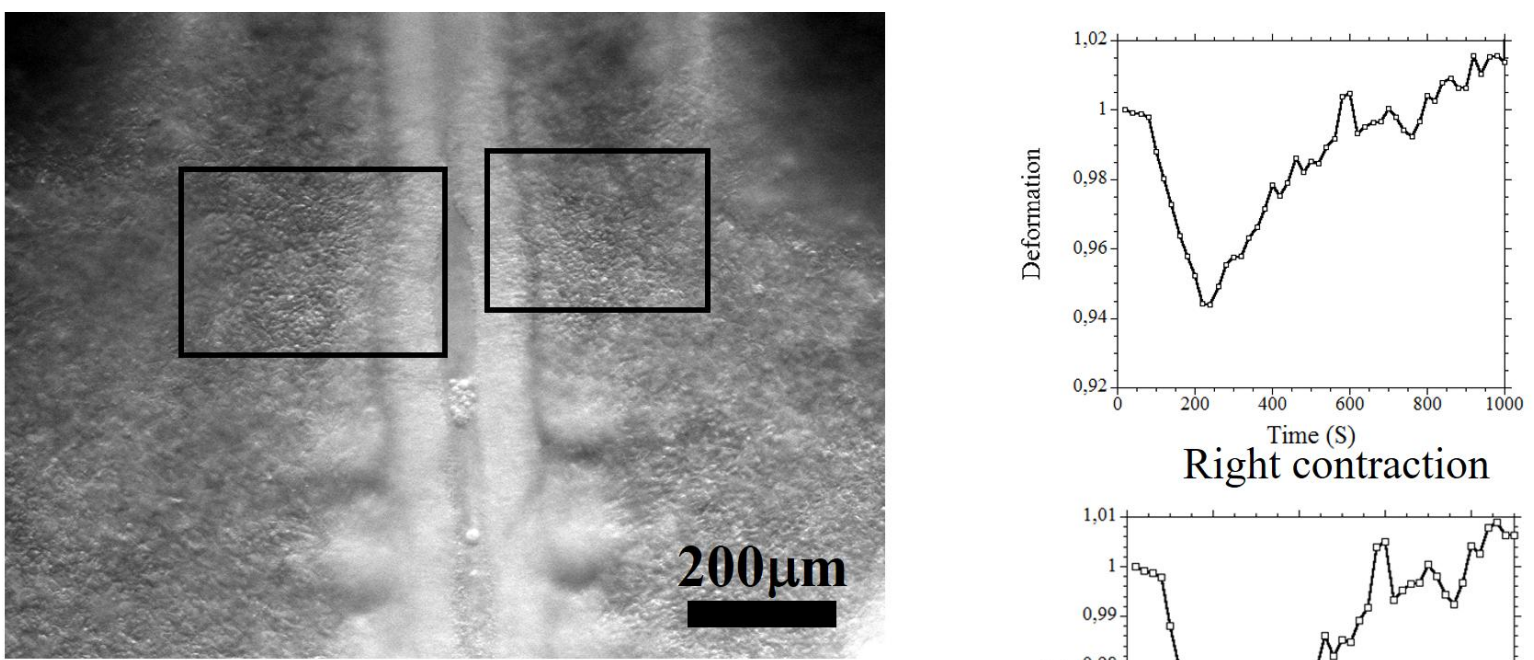

Right contraction

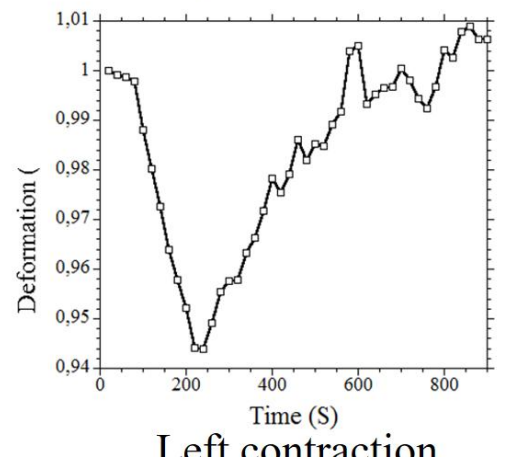

Figure 4D 
Figure 4 Fig. 4A In vivo observation of eye formation shows a sudden superficial contraction generating the eye lens inside an eye cup (from Video 8). The four images to the left show a Time-Lapse video of the phenomenon during 6 hours (Mag. 10X). The two PIV analyses show, first (Top), the contracting ectoderm at an early stage when the contraction of the placode generates a constriction in the orthoradial direction and a thickening in the radial direction (the movement points outwards because of compression). The second PIV analysis shows the subsequent invagination after delamination of the lens ectoderm (the movement points inwards the eye ball because of buckling). The PIV analyses are made at moments corresponding to the Top-Left and Top-Right snapshots. The principle is similar to body formation, except that instead of forming tubes inside tubes, the eye forms a sphere (the lens) inside a sphere (the eye globe). In Fig. 4A Bottom-Right, we show the lens and the globe during formation. The arrowhead points to the line of delamination of the superficial ectoderm which rounds off to form the lens, while the underneath ectoderm invaginates to form the eye globe and retina. The graph in Fig. 4A Bottom-Right, shows the non-linearity of the temporal evolution of the ectoderm which invaginates (the data points obtained by PIV tracking follow the downwards movement of the invaginating ectoderm). Fig. 4B In the ear, it is observed that the contraction exhibits an asymmetry along the DV boundary, which gives to the ear its specific conchoidal form (from Video 6, see also Video 7 for another example). Fig. 4C When the tissue movements are followed with a fine temporal resolution ( 1 frame/10Sec.), it is seen that there exist, since the blastula stage, sporadic contractions having a coherent spatial and temporal pattern. The PIV analysis (Fig. 4C Top-Left) and the temporal variation (Fig. 4C Top-Right) are extracted from Video 10 . They show a $15 \%$ deformation over a 5 Min. contraction. Bottom of Fig. 4C shows the blastula at the time of this twitch (same embryo). The sectors are visible without any staining. Arrowhead points to the eye sector, the bigger arrow points to the mouth sector, and the smaller arrow to the ear sector. Fig. 4D, at a later stage (neurulation, formation of the first somites, Mag. 10X), we still observe sporadic contraction twitches of the presumptive ear territory. The contractions are actually bilateral (the box shows the PIV map of the contractions, the graphs the temporal evolution in the center of the contracting domain). These observations show that there exists an intrinsic contractility of the tissue much earlier than the actual morphogenetic events. Also, the textural geometry governs the spatial pattern of forces, and locks the buckling pattern. 


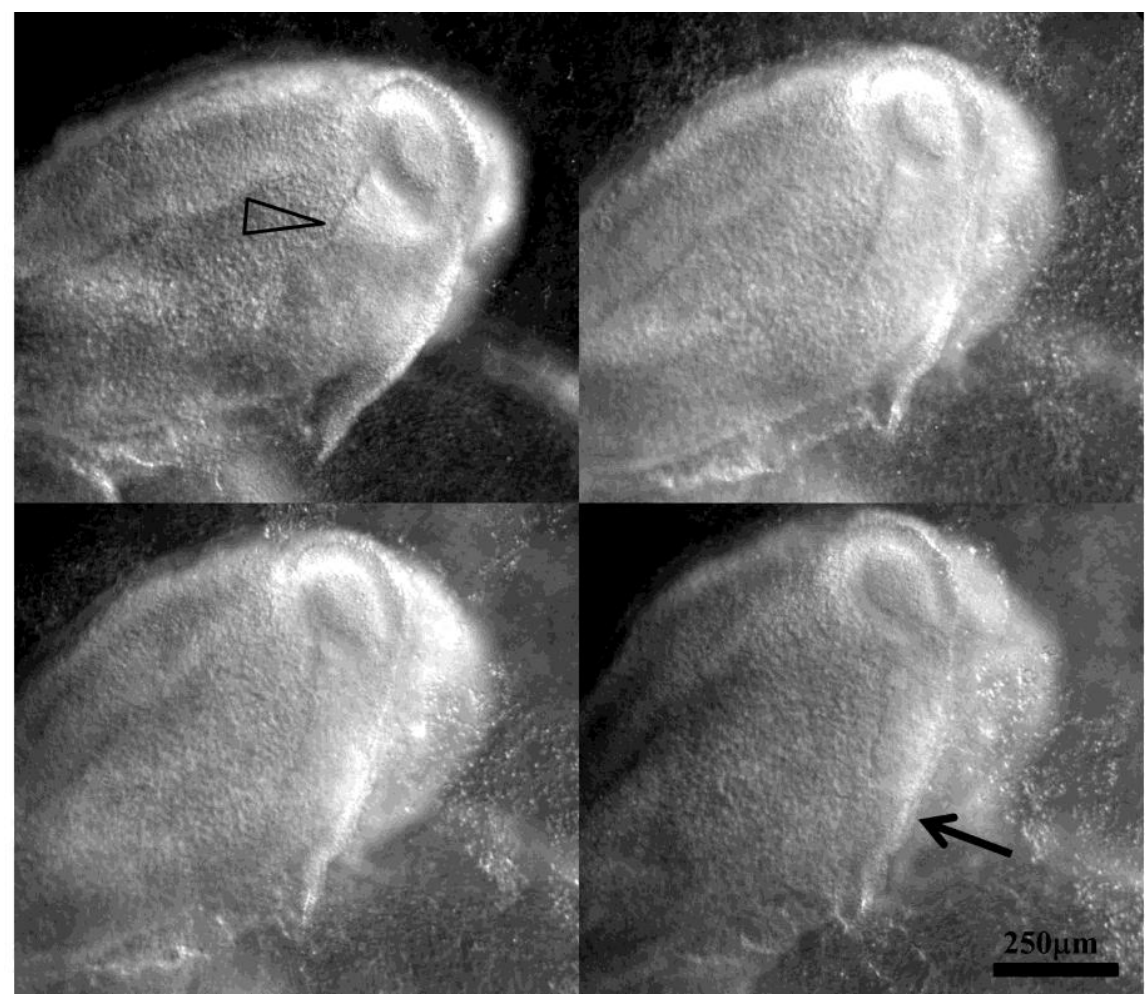

Figure 5A

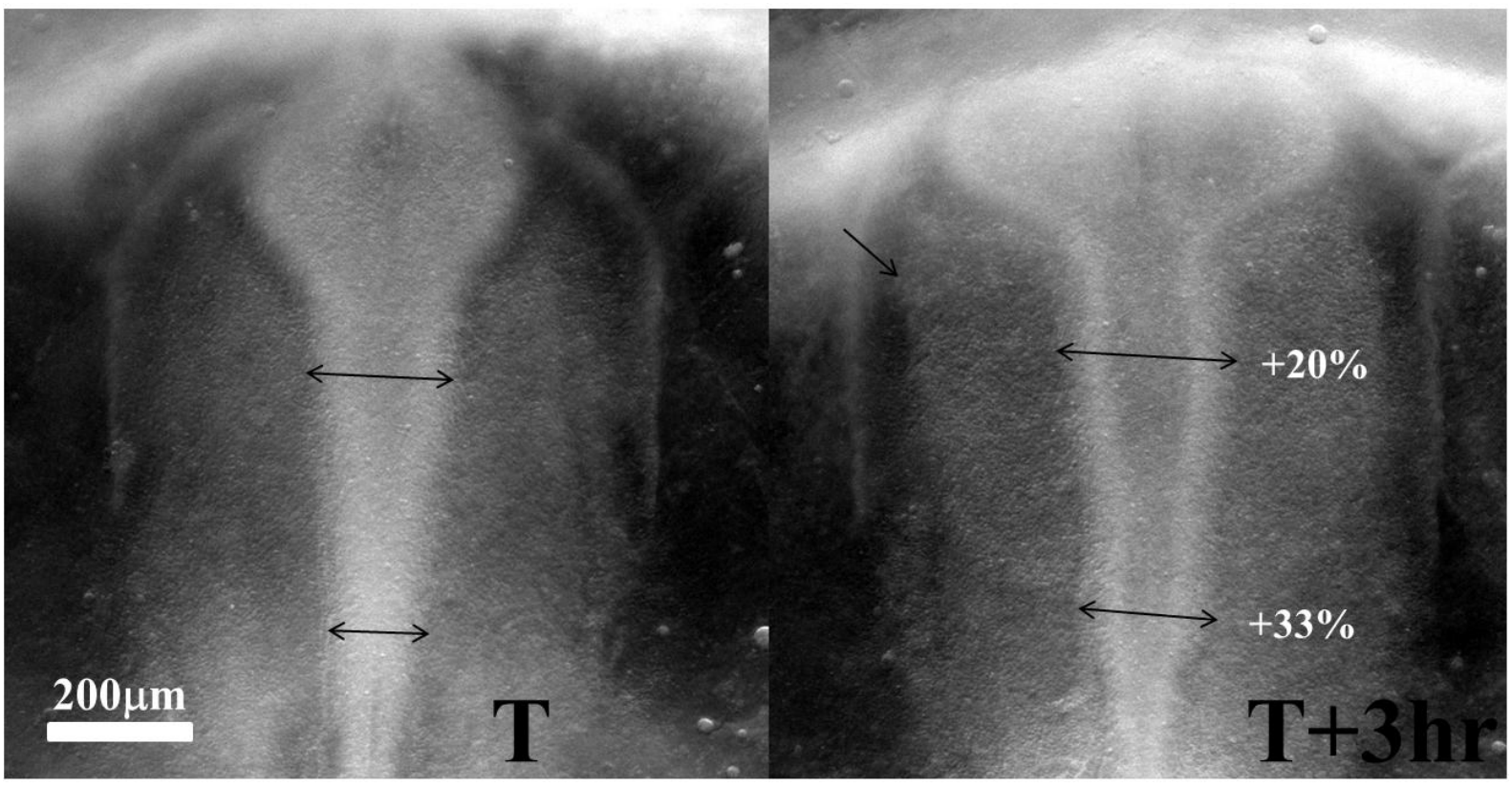




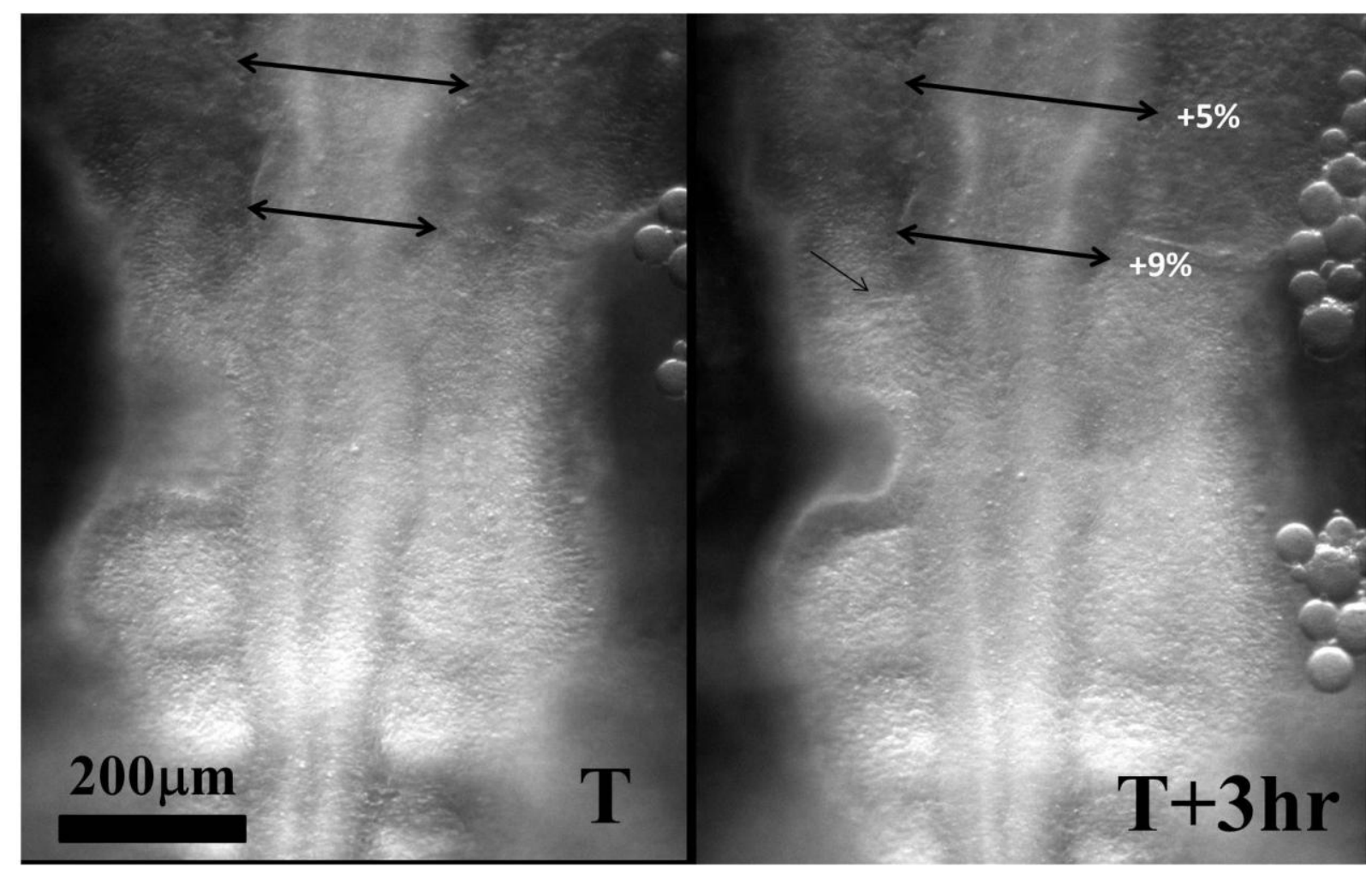

Figure 5B

Figure 5 Observation of the Neural Crest Cells (NCCs). During the morphogenetic movements, NCCs migrate to form bony structures and nerves. We observe quite clearly that the NCCs migrate and swerve around the ocular (Fig. 5A, from Video 11) and otic (Fig. 5B Top, from Video 13) placodes. In Fig. 5A, the straight line emanating from the eye cup (arrow) is actually the very boundary of the eye sector, which forms a wrinkle homolog to the cheek wrinkle on human faces. The continuity between the eye contour and the sector edge is obvious. The migration of NCCs around the placode explains why eye and ear tissue are softer and prone to generating orifices, surrounded by a thicker protective tissue such as brow bones. Fig. 5B shows that the onset of NCCs migration is associated to a shortening and a widening of the neural tube, which finds a simple explanation in the fact that reduction of the number of cells along the neural tube implies a mechanical shortening by conservation (this is symmetrical to elongation when cells intercalate [34]). The top montage shows the onset and propagation of the cranial NCCs (the thin arrow points to the flow of NCCs, from Video 13). The bottom montage shows the onset and flow of otic NCCs, the thin arrow points to the NCCs flux which is directly visible without any staining. 


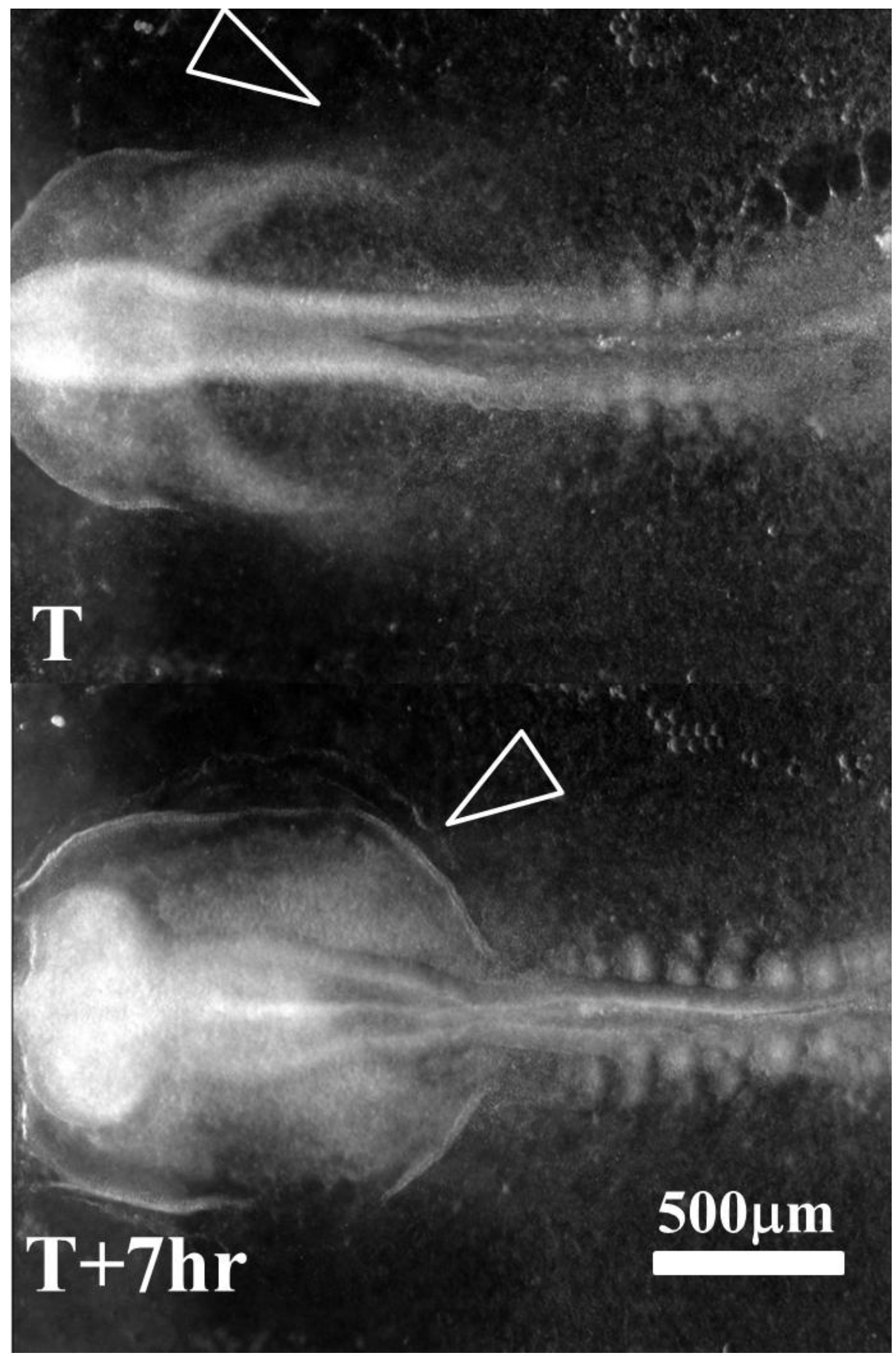

Figure 6 
Figure 6 When preparing embryos for optical microscopy, we have to cut them off the vitelline membrane. This reduces the peripheral tension around the embryo. We often observe a premature buckling of the ear sector (arrowhead), in the form of a hairpin instead of an orifice (from Video 14).

\section{2}

\section{Embryo 1 Embryo 2}

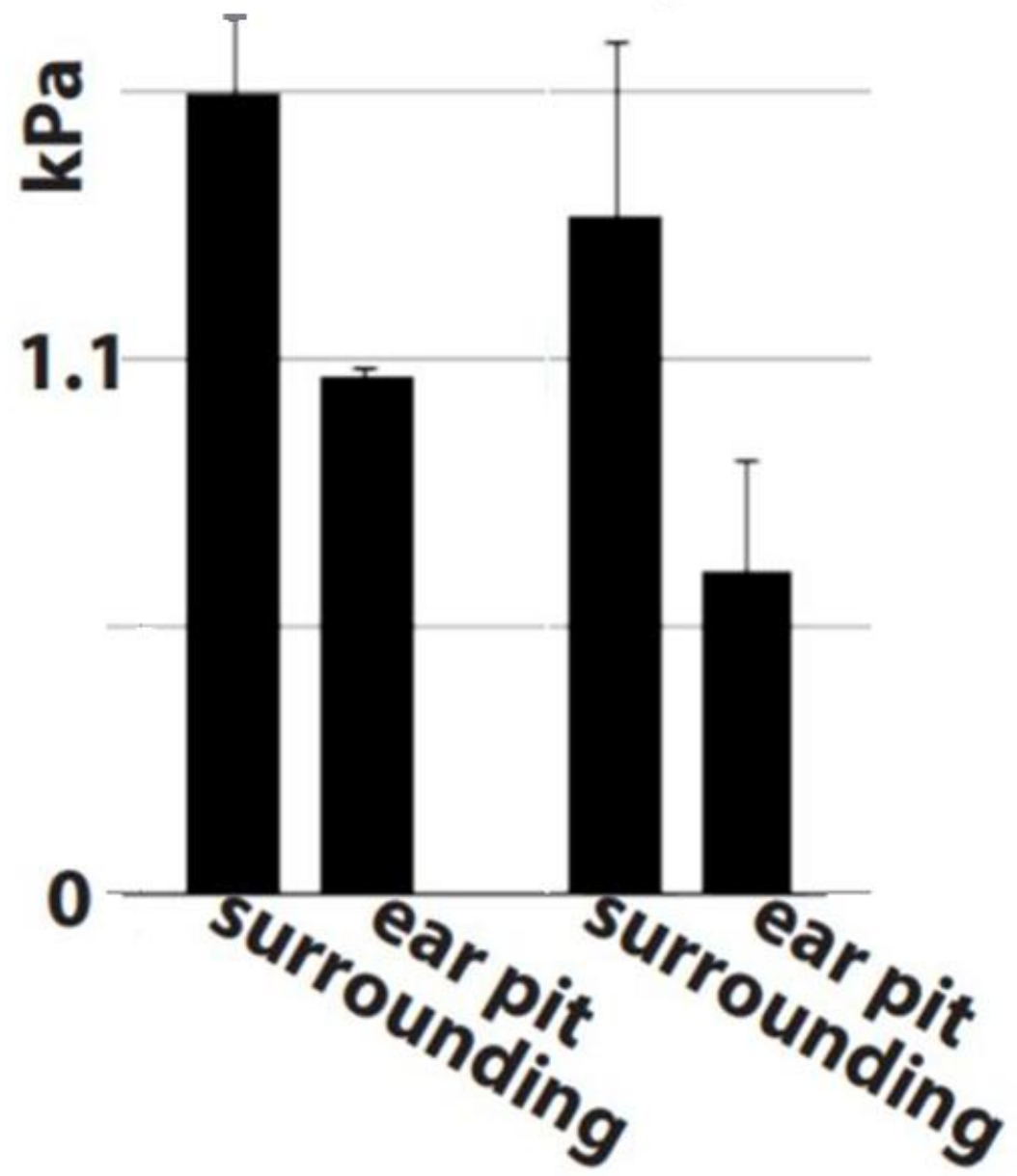

Figure 7A 


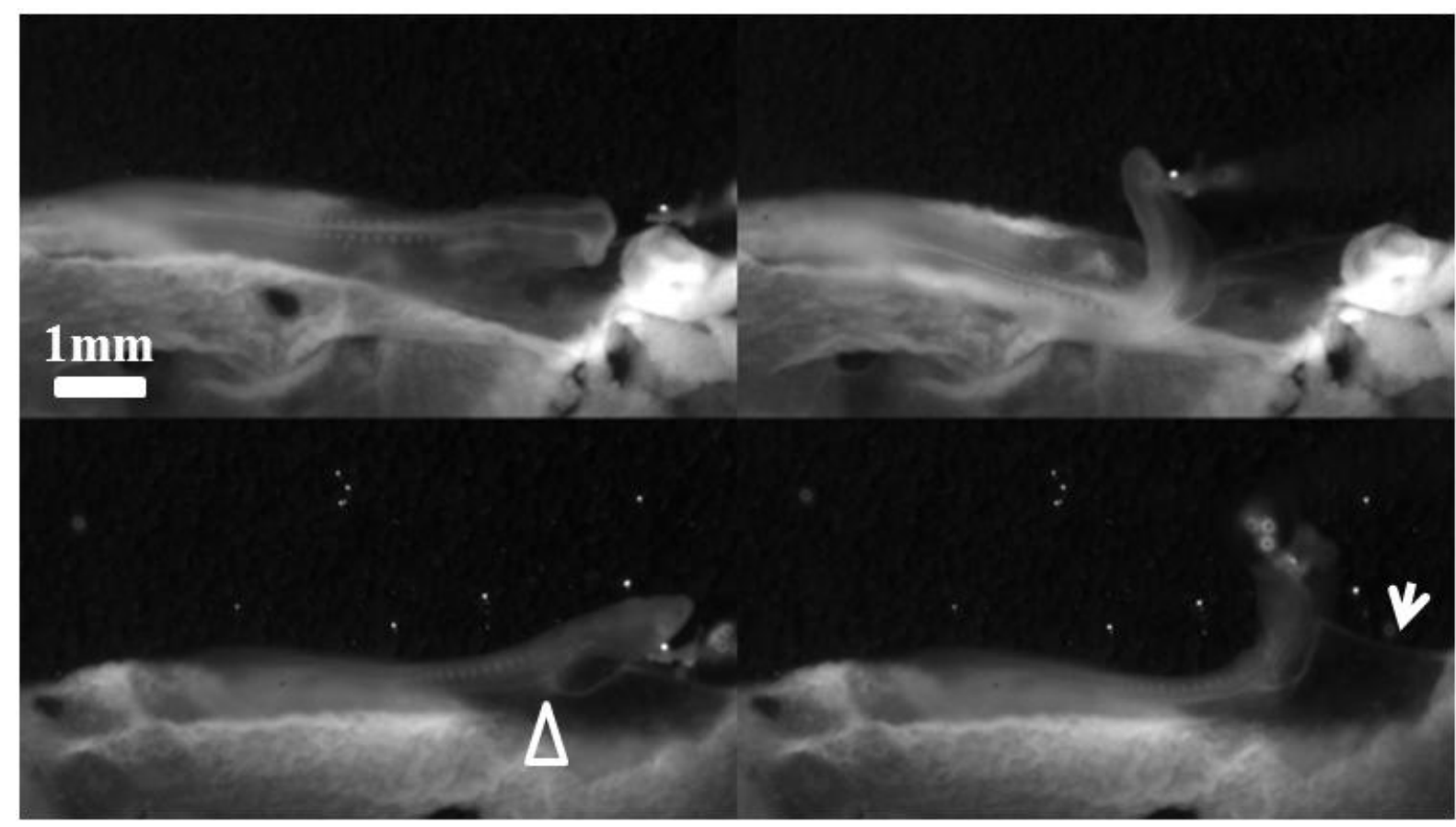

Figure 7B

Figure 7 Fig. 7A We assessed the elasticity of the tissue by AFM. Measurements at HH stage 9-10 (prior to actual ear pit formation). AFM measurements in force spectrosopy mode reveal a softer tissue in the presumptive ear pit, as compared to the tissue around the future ear pit. The elasticy difference just prior to ear pit formation shows an elastic modulus $\sim 0.75 \mathrm{kPa}$ in the presumptive ear pit, as compared to $\sim 1.5 \mathrm{kPa}$ in the surrounding tissue. Fig. 7B We also flexed embryos at stages $\mathrm{HH} 10$ and 11 in Fig. 7A, with motorized glass pipettes. We observe that when a force is applied perpendicularly to the embryo AP axis, the embryo flexes in the presumptive ear area (and even buckles inwards in this area), confirming that this area is softer. 


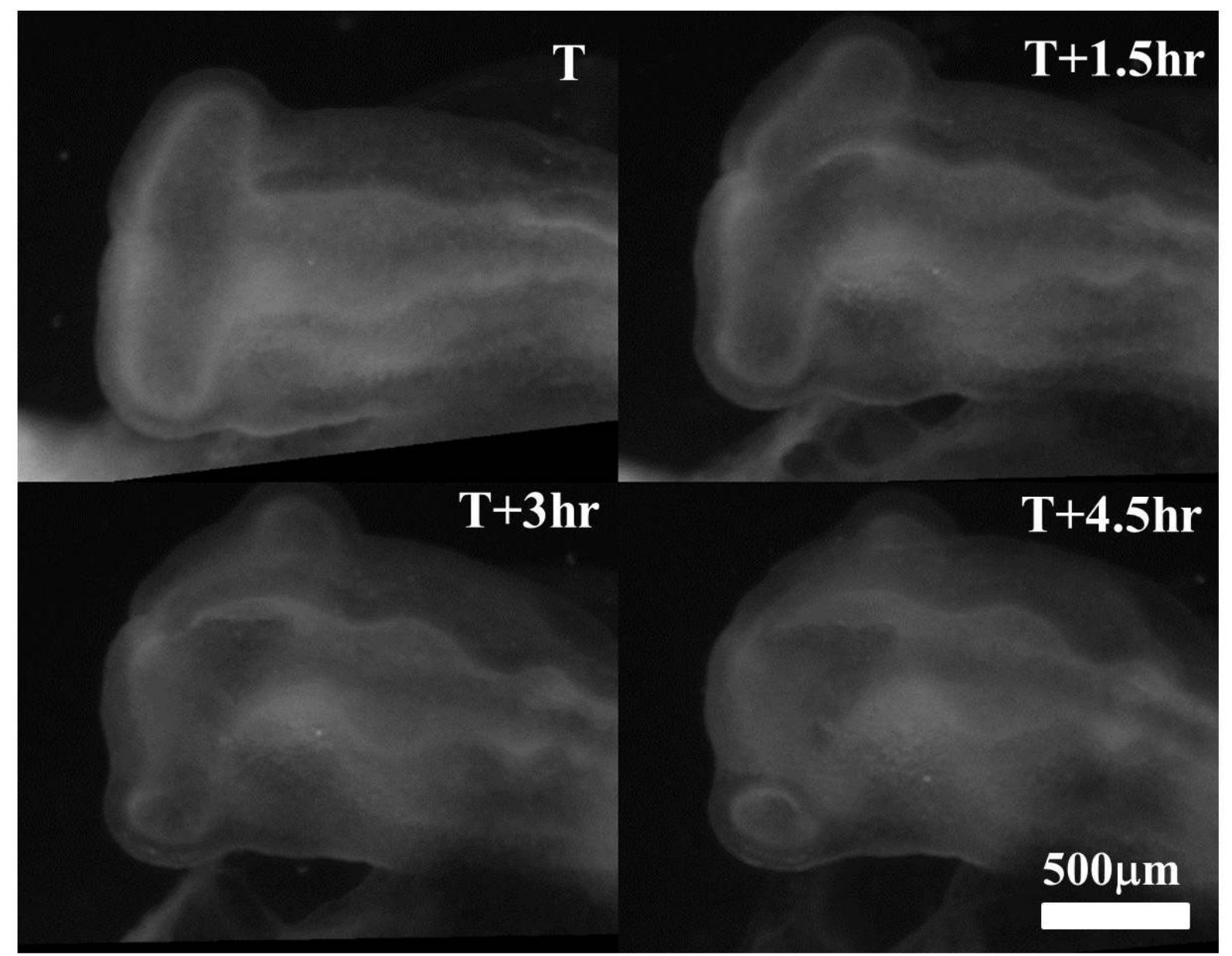

Figure 8A
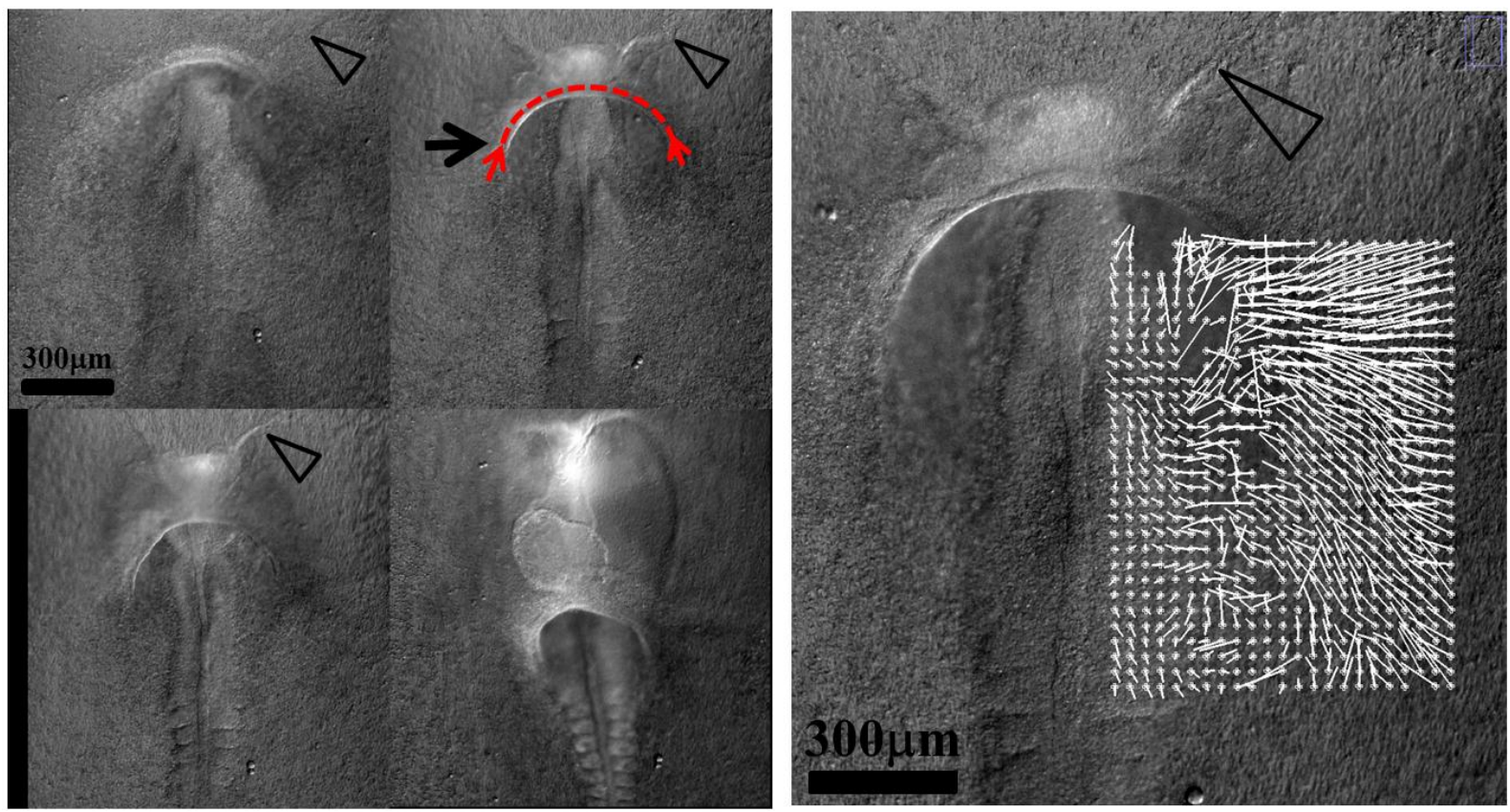

Figure 8B 

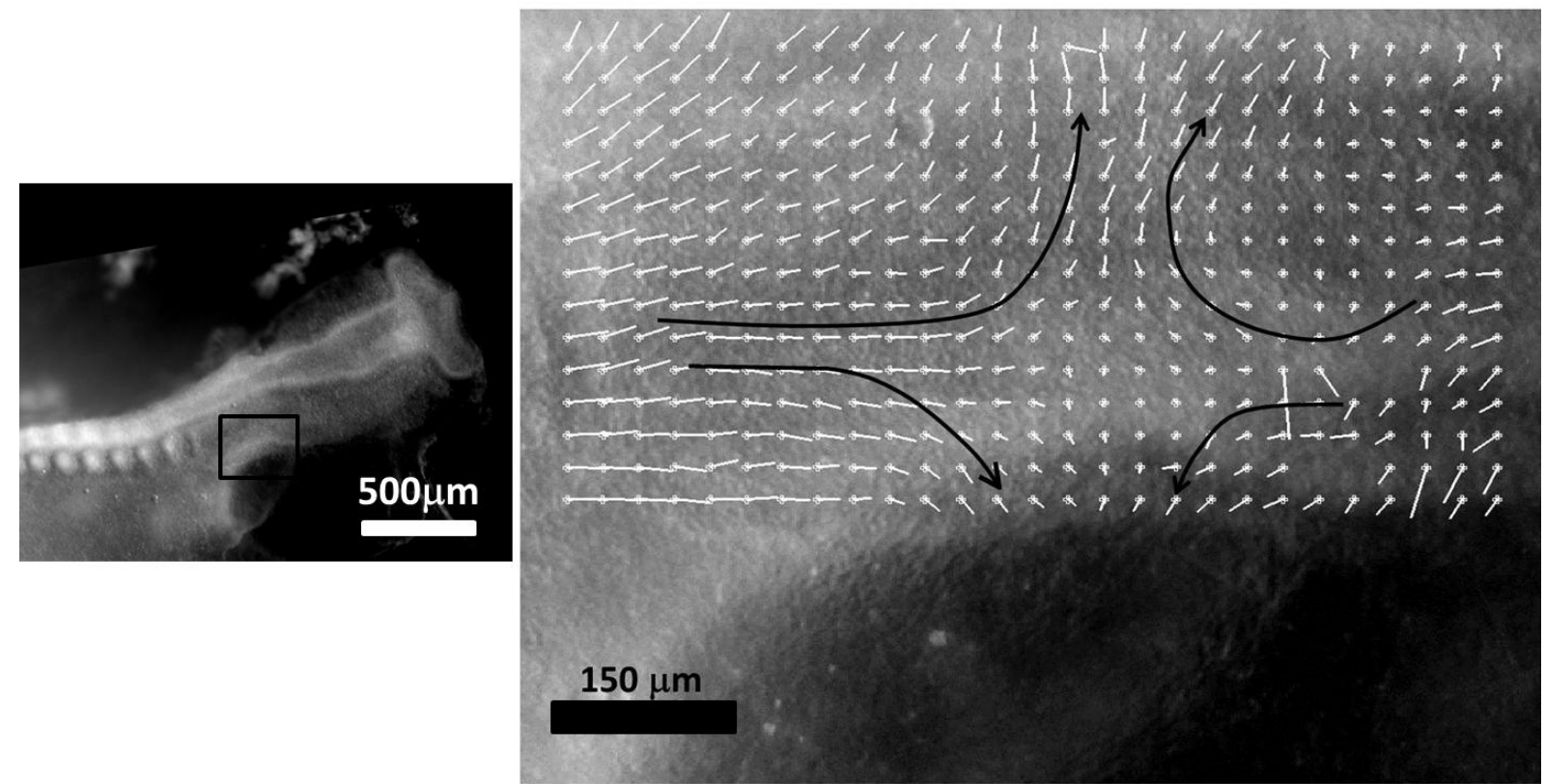

Figure 8C

Figure 8 Morphogenesis of eyes and ears implies considerable movements. Eye movement is simpler to understand, the eye stalk extending in a hammer shape locked onto the eye sector and the eye cup colliding against the ectoderm where the placode rounds off under compression (Fig. 8A, from Video 15). Ear movements are more difficult to understand because there is shear. In addition to the movements described in the literature $[7,9,16]$, there exists an underneath dipolar pull by the heart crescent (Fig. 8B), and a quadrupolar winding of the ear territory due to contraction along the DV boundary (Fig. 8C). Fig. 8B Shows four snapshots on the ventral side of the embryo, during establishment of the ear territory. When filmed from underneath, the Time-Lapse microscopy reveals a very important shear of the body tissue by the heart and gut crescent (Arrow), the force is oriented along the purse string formed by the edge (dots). Arrowheads point to the quite visible boundary of the ocular sector. During this shear the tissue is dragged towards the cardiac crescent, and the mesodermal cells are sipped into the cardiac area. Fig. $8 \mathrm{C}$ shows the PIV analysis of the contraction during 10 minutes of movement, in the area of the ear territory of a day 2 embryo, $\mathrm{HH}$ stage 11 (vectors rescaled to visible sizes). It shows a quadrupolar contraction winding the ear territory towards the dorsal area. 
38
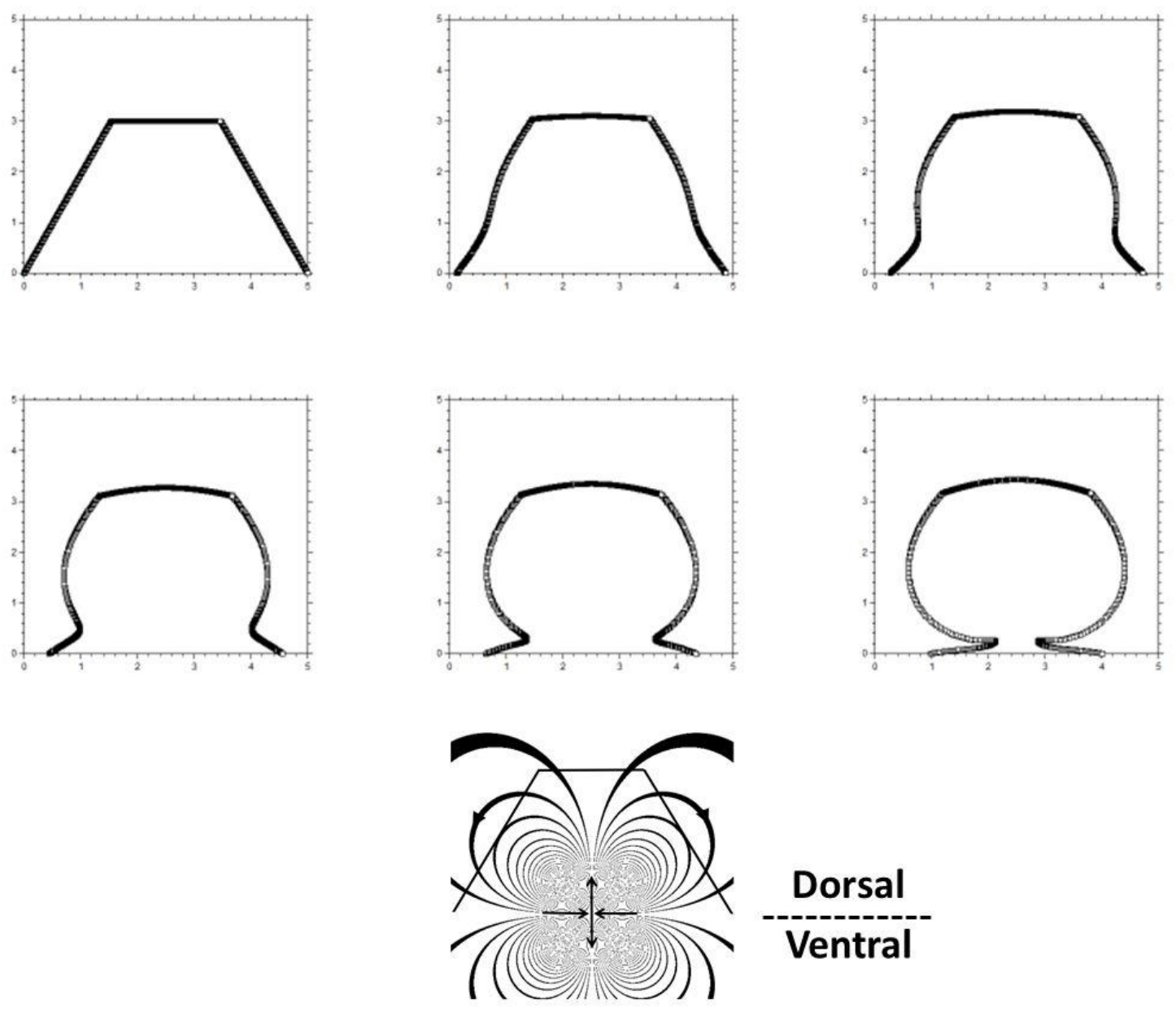

Figure 9A 

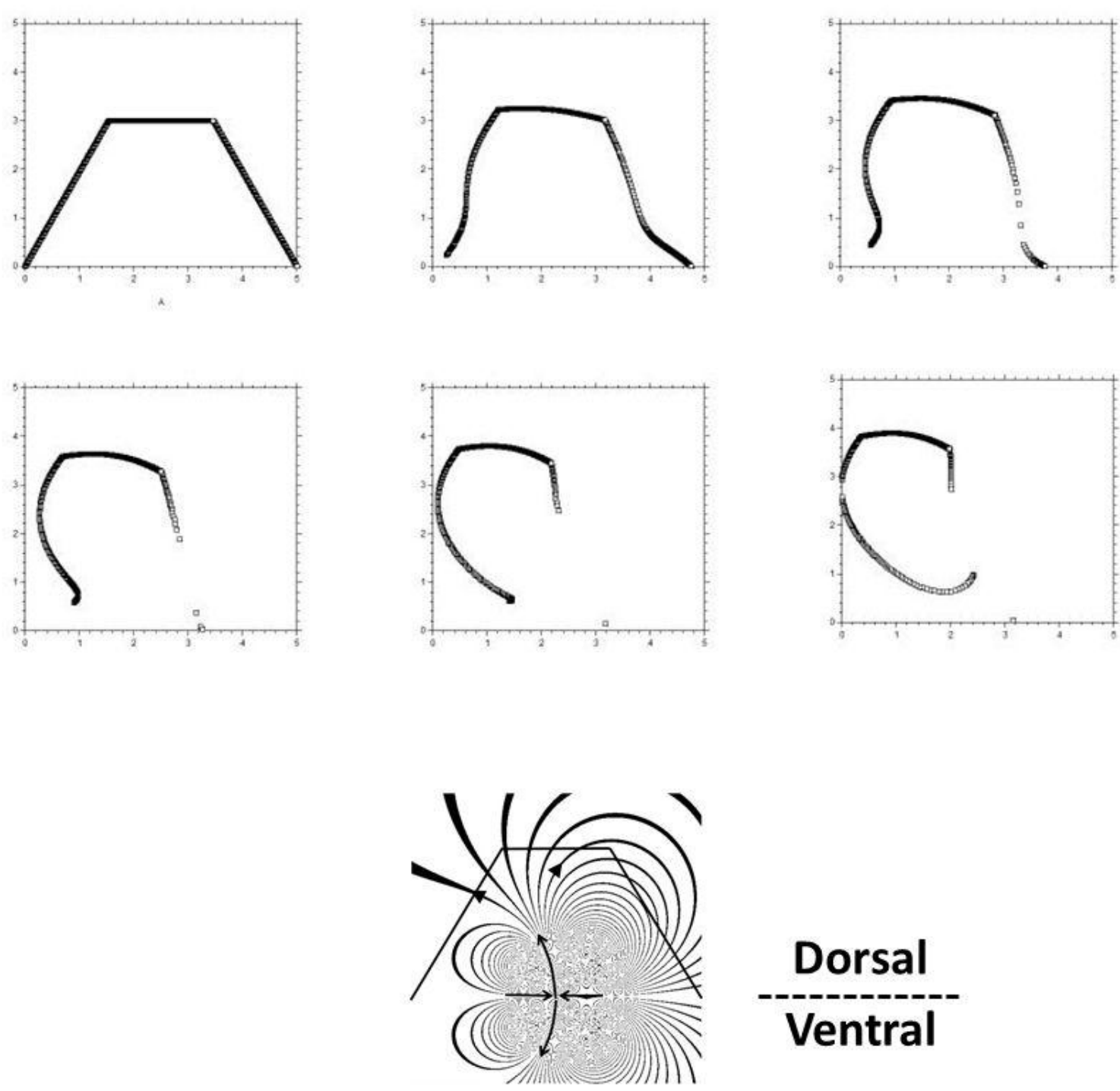

\section{Dorsal}

\section{Ventral}

Figure 9B

Figure 9 Modelling of movements using the analytics developed in Ref. 8. We start with a truncated sector describing the otic territory (Fig. 9A), and assume a quadrupolar contraction along the horizontal axis at the DV boundary located at $y=0$. The flow is calculated analytically around four logarithmic vortices [8] forming a quadrupolar vortex flow. For the calculations in Fig. $9 a=2, c=3, b=1$. For the calculation in Fig. $9 A, \alpha=\beta$, for the calculation in Fig. 9B $\beta=2 \alpha$. The iso- $\Psi(x, y)$ map shows the associated streamline pattern. The trapezoidal kink progressively rounds off. Fig. 9B If we introduce an asymmetrical quadrupole we generate very simply conchoidal forms corresponding to ear patterns. 

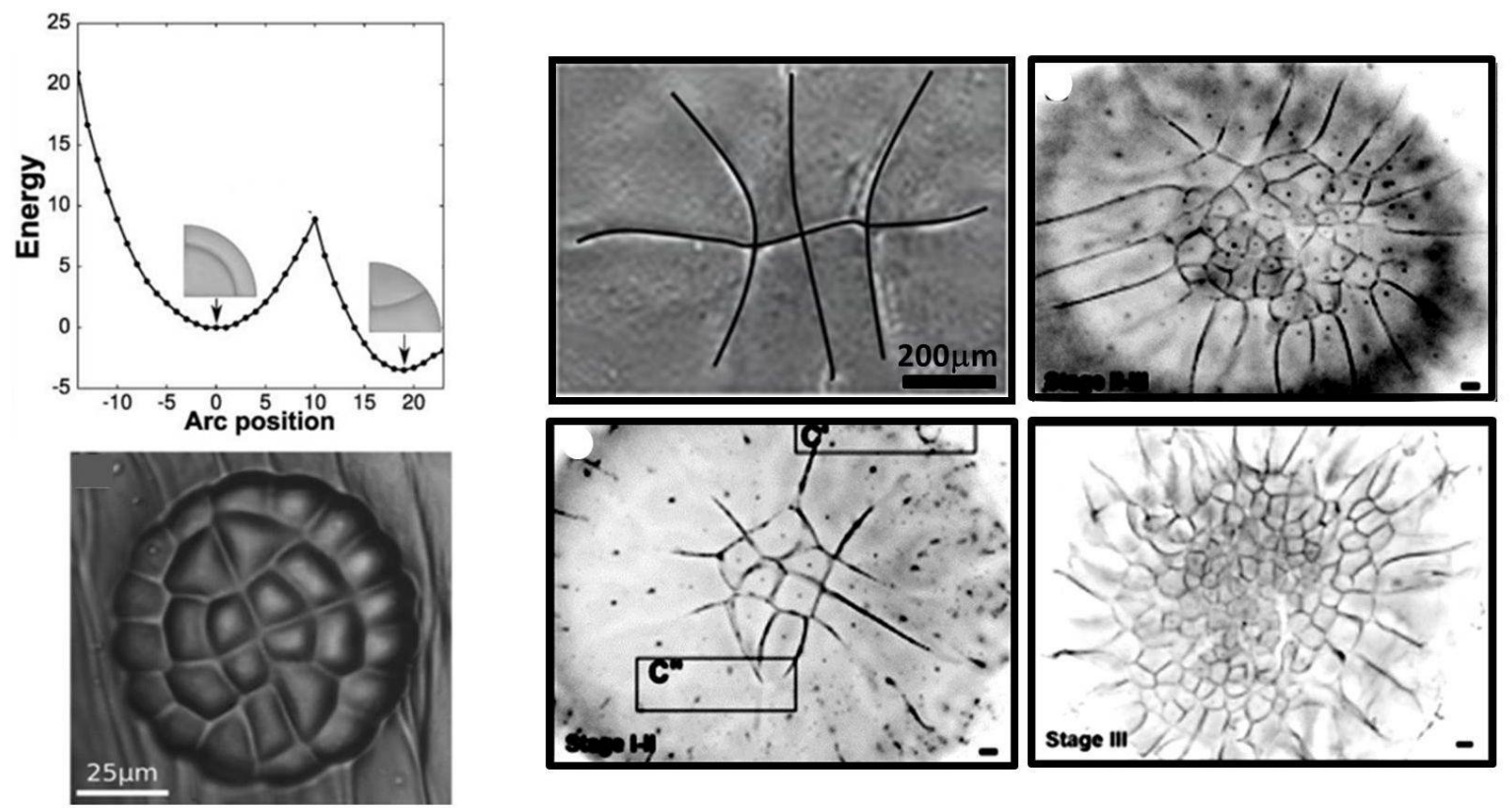

Figure 10A

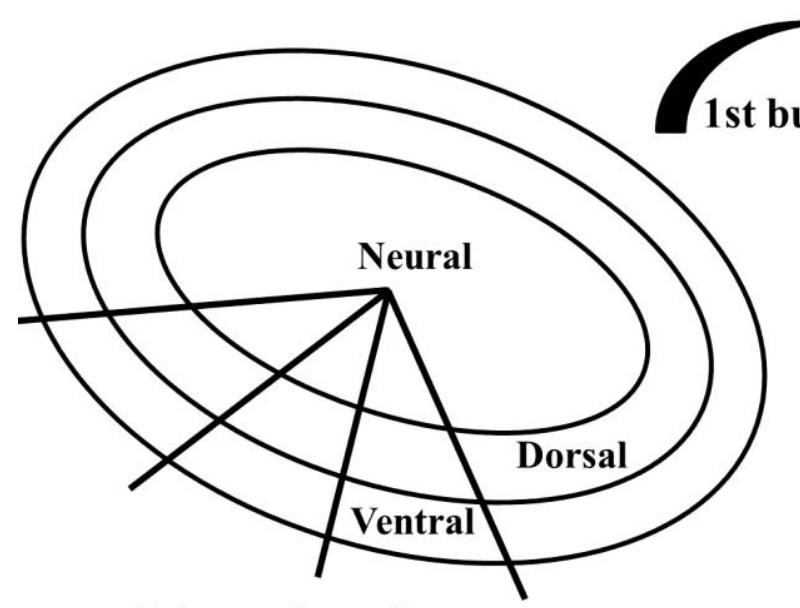

Extra-embryonic

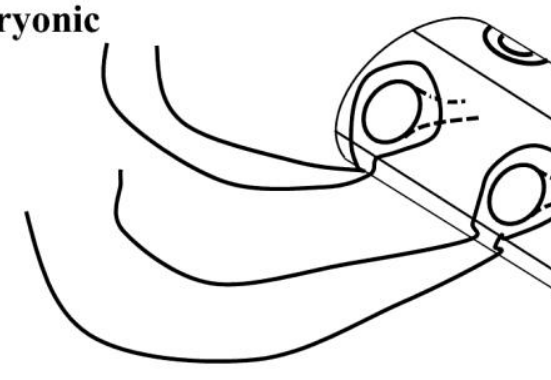

Extra-embryonic
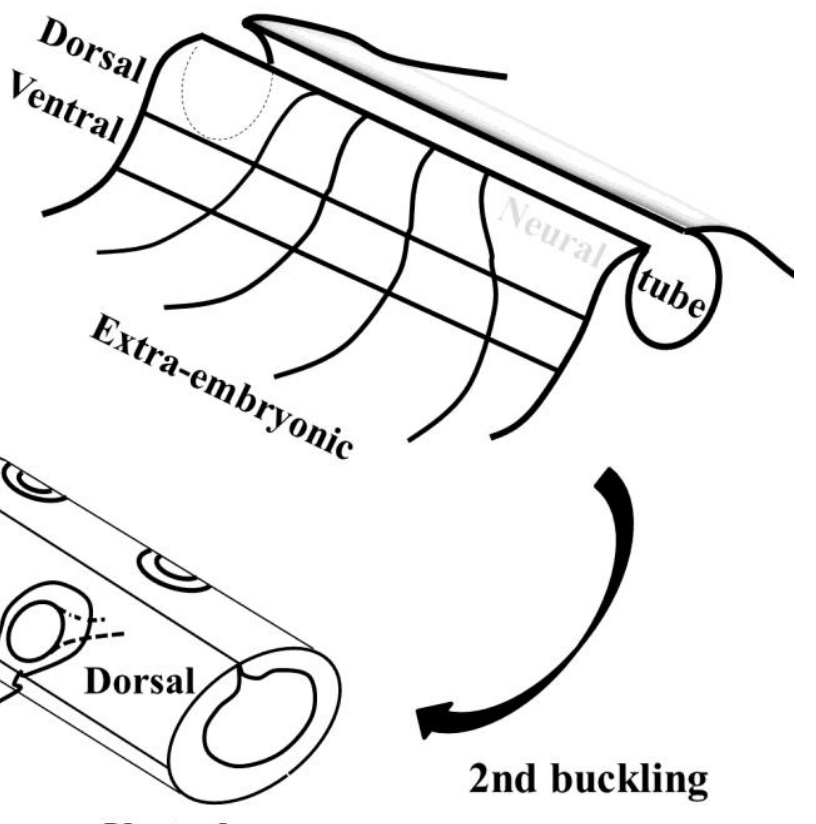

Ventral 


\section{Figure 10B}

Figure 10 Fig. 10 A Top-Left : The origin of the structure in rings and sectors lies in the fact that radial cleavage is the most favorable, and orthoradial cleavage is the next most favorable cleavage (adapted from [24]). Bottom-Left : In plants the radial and orthoradial cleavages are very robust and conspicuous. Fig. 10A Right. Four stages of early cleavage in chicken, adapted from Refs. 20 and 21. In chicken, the first cleavages follow a pattern of radial and orthoradial cleavages which is arguably less conspicuous than in plants. Please note that the lines of third cleavage are nevertheless identical in Chicken and in Dionaea Muscipula. Fig. 10B A system of intersected rings and sectors undergoing biaxial tension and buckling will self deform into a system consisting of tubes inside tubes, decorated at right angles by tubular orifices diving down towards the neural tube. 